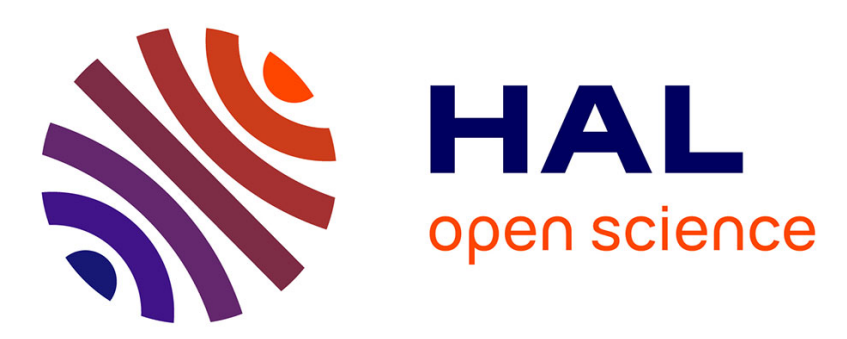

\title{
Non-linear galloping of sagged cables in 1:2 internal resonance
}

Angelo Luongo, Giuseppe Piccardo

\section{To cite this version:}

Angelo Luongo, Giuseppe Piccardo. Non-linear galloping of sagged cables in 1:2 internal resonance. Journal of Sound and Vibration, 1998, 214 (5), pp.915-940. hal-00812553

\section{HAL Id: hal-00812553 https://hal.science/hal-00812553}

Submitted on 12 Apr 2013

HAL is a multi-disciplinary open access archive for the deposit and dissemination of scientific research documents, whether they are published or not. The documents may come from teaching and research institutions in France or abroad, or from public or private research centers.
L'archive ouverte pluridisciplinaire HAL, est destinée au dépôt et à la diffusion de documents scientifiques de niveau recherche, publiés ou non, émanant des établissements d'enseignement et de recherche français ou étrangers, des laboratoires publics ou privés. 


\author{
A. LUONGO \\ Dipartimento di Ingegneria delle Strutture, delle Acque e del Terreno, \\ Università degli Studi di L'Aquila, 67040 Monteluco di Roio, L'Aquila, Italy \\ AND

\section{G. PICCARDo} \\ Dipartimento di Ingegneria Strutturale e Geotecnica, Università degli Studi di Genova, \\ via Montallegro 1, 16145 Genova, Italy
}

The aeroelastic behaviour of a flexible elastic suspended cable driven by mean wind speed, blowing perpendicularly to the cable's plane, is investigated. By applying the Galerkin procedure to the partial differential equations of motion and using an in-plane and an out-of-plane mode as shape functions, a two-d.o.f. model is derived. The discrete equations are coupled through quadratic and cubic terms arising both from geometric and aerodynamic effects. The associated linear frequencies are assumed to be in an almost 1:2 ratio, so that internal resonance occurs. The multiple scale perturbation method is employed to obtain a set of three amplitude modulation equations, whose coefficients depend on the mean wind speed, which is assumed as control parameter. Two perturbative solutions are developed, each based on a different assumption about the order of magnitude of the static displacements, produced by steady state wind forces. Analytical results are then compared with direct numerical integrations of discrete non-linear equations. By performing a bifurcation analysis, the existence of several equilibrium branches is proved. The relative importance of geometric and aerodynamic non-linearities is discussed through simplified models. The influence on critical and postcritical behaviour of several parameters, including geometrical cable parameters, detuning and non-symmetric flow effects, is investigated. The important role played by the steady state forces is highlighted.

\title{
1. INTRODUCTION
}

Iced cables can experience self-excited aeroelastic oscillations of large amplitude due to wind, mainly in the vertical plane. The problem has been widely studied in the literature for slender beams or taut strings, both in linear and non-linear fields, by using simple models with one or two-d.o.f. [1, 2]. Large galloping oscillations of suspended cables have only recently been analyzed for a three-d.o.f. system [3]. In these works, however, only the aerodynamic non-linearities have been taken into account, while geometric non-linearities have been ignored (e.g. see reference [3], where displacements up to only second order have been retained in the strain energy). However, geometric effects play an important role in describing the dynamic behaviour of cables, as already highlighted in many works on the subject; see, e.g., references [4-6]. In particular, there is a strong coupling between in-plane and out-of-plane motion when internal resonance conditions occur. So it seems important to propose a new model in which both geometric and aerodynamic non-linearities are considered. 
In this paper a study is presented of the response of a flexible elastic suspended cable in internal resonance condition of $1: 2$ type, excited by a wind flow perpendicular to the plane of the cable. Preliminary results on the subject have been presented by the authors in reference [7]. The wind loads are determined in a quasi-static regime by considering translational displacements only. Torsional effects are neglected since single cables usually have a torsional frequency much higher than two flexural frequencies [8]. The aerodynamic forces are assumed to be acting in the plane containing the cable cross-section in the reference equilibrium configuration; therefore, the effects due to the change of geometry and the longitudinal component of the forces are neglected. To analyze coupling phenomena between in-plane and out-of-plane motions, a simple two-d.o.f. model is derived from the continuous one via the Galerkin procedure, by taking one modal shape in each plane. The cable is assumed to be at the first "cross-over" point [9], so that linear frequencies are almost in a 1:2 ratio. The discrete non-linear equations of motion are studied through the multiple scales perturbation method. Particular attention is devoted to the problem of how to order correctly, in the perturbation scheme, the displacements produced by the steady state wind forces. Two assumptions are made about the order of magnitude of such static displacements, each leading to a set of amplitude modulation equations with different coefficients. The validity of these assumptions is then discussed by comparing analytical and numerical results. A bifurcation analysis of the amplitude equilibrium paths reveals a very rich postcritical behaviour of the cable. Moreover, an extensive parametric analysis makes it possible to discuss the reliability of simplified models in which geometrical non-linearities, aerodynamic non-linearities and/or steady state wind forces are in turn neglected.

\section{EQUATIONS OF MOTION}

Consider a heavy hyperelastic cable suspended between two fixed supports. The equations governing the planar motion have been developed in reference [4], in Cartesian co-ordinates; subsequently, they have been extended to three-dimensional motion, referred to Frenet $[5,10]$ or Cartesian [11] triads. Here, the reference is to the Frenet triad in which the aerodynamic forces can be more easily expressed. Let $u_{i}(i=1,2,3)$ be the displacement components measured from the static equilibrium configuration (see Figure 1). The dimensionless equations that govern the prevalently transversal motion of the cable in the small curvature regime are [5, 12]:

$$
[1+\alpha e] \tilde{u}_{1}^{\prime \prime}+\beta \tilde{f}_{1}=\pi^{2} \tilde{u}_{1}, \quad[1+\alpha e] \tilde{u}_{2}^{\prime \prime}+\alpha \beta e+\beta \tilde{f}_{2}=\pi^{2} \tilde{u}_{2},
$$

where

$$
e=\left(\tilde{u}_{3}^{\prime}-\beta \tilde{u}_{2}\right)+\frac{1}{2}\left[\tilde{u}_{2}^{\prime 2}+\tilde{u}_{1}^{\prime 2}\right]
$$

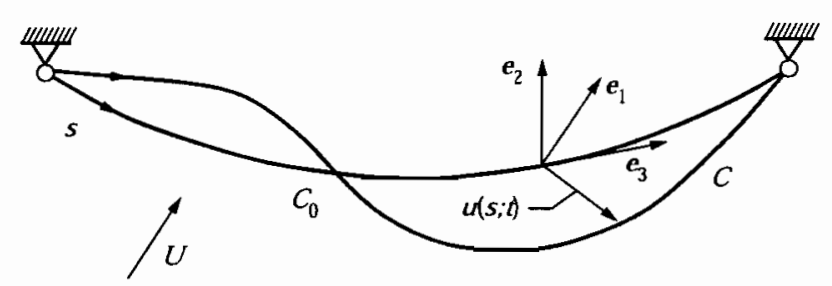

Figure 1. Cable configuration: $C_{0}$ equilibrium configuration, $C$ dynamic configuration; $e_{i}(i=1,2,3)$ binormal, normal and tangential unit vectors; $U$ uniform wind velocity. 
By expanding $\gamma_{a}$ and $V$ in power series of the velocity components and retaining up to third order terms, the following expressions for the non-dimensional forces are obtained:

$$
f_{i}=f_{i 0}+f_{i 1} \dot{u}_{1}+f_{i 2} \dot{u}_{2}+f_{i 3} \dot{u}_{1}^{2}+f_{i 4} \dot{u}_{2}^{2}+f_{i s} \dot{u}_{1} \dot{u}_{2}+f_{i 6} \dot{u}_{2}^{3}, \quad i=1,2 .
$$

In equation (5) coefficients $f_{i j}(j=1, \ldots, 6)$ depend on the drag and lift coefficients together with their derivatives; in addition, some of them also depend on the dimensionless uniform wind velocity,

$$
\mu=(\rho b U / m) \sqrt{L / g},
$$

which $\mu$ is assumed to be the control parameter. The binormal force $f_{10}$ and the normal force $f_{20}$ are driven exclusively by the mean wind speed; they will be referred to as steady state forces.

By neglecting the effects due to cable flexibility, the forces (5) acting on the rigid cylinder are directly applied to the cable in the normal (lift force, $f_{2}$ ) and binormal (drag force, $f_{1}$ ) direction; consequently, no forces are considered in the tangential direction $\left(f_{3}=0\right)$.

\section{DISCRETE MODEL}

A discrete two-d.o.f. model is obtained from equations (1). The two components of motion are assumed as:

$$
u_{i}(s ; t)=q_{i}(t) \phi_{i}(s), \quad i=1,2,
$$

where $q_{i}(t)$ describe the temporal behaviour and $\phi_{i}(s)$ are the out-of-plane $(i=1)$ and in-plane $(i=2)$ modes, respectively, taken from the linear theory [9]. By applying the Galerkin method, two ordinary differential equations are obtained:

$$
\begin{gathered}
\ddot{q}_{1}+b_{1} \dot{q}_{1}+b_{2} \dot{q}_{2}+\omega_{1}^{2} q_{1}+c_{1} q_{1} q_{2}+c_{2} q_{1}^{3}+c_{3} q_{1} q_{2}^{2}+b_{5} \dot{q}_{1}^{2}+b_{6} \dot{q}_{2}^{2}+b_{7} \dot{q}_{1} \dot{q}_{2}+b_{8} \dot{q}_{2}^{3}=f_{10}, \\
\ddot{q}_{2}+b_{3} \dot{q}_{1}+b_{4} \dot{q}_{2}+\omega_{2}^{2} q_{2}+c_{4} q_{1}^{2}+c_{5} q_{2}^{2}+c_{6} q_{1}^{2} q_{2}+c_{7} q_{2}^{3}+b_{9} \dot{q}_{1}^{2}+b_{10} \dot{q}_{2}^{2}+b_{11} \dot{q}_{1} \dot{q}_{2} \\
+b_{12} \dot{q}_{2}^{3}=f_{20} .
\end{gathered}
$$

In equations (8) $\omega_{1}$ and $\omega_{2}$ are non-dimensional natural frequencies; due to the dimensionless time in equations (3), they are equal to the ratios between the dimensional frequencies of the two modes considered and the lower out-of-plane frequency of the linear cable. Both quadratic and cubic non-linearities appear in the equations of motion. The coefficients of equations (8) are defined in Appendix A; details can be found in reference [12].

Equations (8) admit non-trivial static solutions $q_{i}=\bar{q}_{i}(i=1,2)$ which satisfy the following non-linear algebraic equations:

$$
\begin{gathered}
\omega_{1}^{2} \bar{q}_{1}+c_{1} \bar{q}_{1} \bar{q}_{2}+c_{2} \bar{q}_{1}^{3}+c_{3} \bar{q}_{1} \bar{q}_{2}^{2}=f_{10}, \\
\omega_{2}^{2} \bar{q}_{2}+c_{4} \bar{q}_{1}^{2}+c_{5} \bar{q}_{2}^{2}+c_{6} \bar{q}_{1}^{2} \bar{q}_{2}+c_{7} \bar{q}_{2}^{3}=f_{20} .
\end{gathered}
$$

Since the $b$ and $c$ coefficients depend on $\mu$, equations (9) implicitly define the equilibrium paths $\bar{q}_{i}=\bar{g}_{i}(\mu)$. Attention will be focused on the (natural) equilibrium path for which displacements $\bar{q}_{i}$ vanish when $\mu=0$; i.e., $\bar{q}_{i}(0)=0$.

In order to separate the effects of the static forces, the displacements $q_{i}(t)$ are expressed as

$$
q_{i}(t ; \mu)=\bar{q}_{i}(\mu)+v_{i}(t ; \mu), \quad i=1,2,
$$


where $v_{i}$ are the dynamic (sliding) displacements measured from the static $\mu$-dependent configuration.

By substituting equations (10) into the equations of motion (8) and accounting for the static equilibrium equations (9), the following non-linear differential equations in the sliding displacements are found:

$$
\begin{aligned}
& \ddot{v}_{1}+k_{1} v_{1}+k_{2} v_{2}+b_{1} \dot{v}_{1}+b_{2} \dot{v}_{2}+c_{1}^{*} v_{1} v_{2}+c_{2} v_{1}^{3}+c_{3} v_{1} v_{2}^{2}+h_{1} v_{1}^{2}+h_{2} v_{2}^{2} \\
& +b_{5} \dot{v}_{1}^{2}+b_{6} \dot{v}_{2}^{2}+b_{7} \dot{v}_{1} \dot{v}_{2}+b_{8} \dot{v}_{2}^{3}=0 \\
& \ddot{v}_{2}+k_{3} v_{1}+k_{4} v_{2}+b_{3} \dot{v}_{1}+b_{4} \dot{v}_{2}+c_{4}^{*} v_{1}^{2}+c_{5}^{*} v_{2}^{2}+c_{6} v_{1}^{2} v_{2}+c_{7} v_{2}^{3}+h_{3} v_{1} v_{2} \\
& +b_{9} \dot{v}_{1}^{2}+b_{10} \dot{v}_{2}^{2}+b_{11} \dot{v}_{1} \dot{v}_{2}+b_{12} \dot{v}_{2}^{3}=0 .
\end{aligned}
$$

Here

$$
\begin{gathered}
k_{1}=\omega_{1}^{2}+c_{1} \bar{q}_{2}+3 c_{2} \bar{q}_{1}^{2}+c_{3} \bar{q}_{2}^{2}, \quad k_{2}=c_{1} \bar{q}_{1}+2 c_{3} \bar{q}_{1} \bar{q}_{2}, \quad k_{3}=2 c_{4} \bar{q}_{1}+2 c_{6} \bar{q}_{1} \bar{q}_{2}, \\
k_{4}=\omega_{2}^{2}+2 c_{5} \bar{q}_{2}+c_{6} \bar{q}_{1}^{2}+3 c_{7} \bar{q}_{2}^{2}, \quad c_{1}^{*}=c_{1}+2 c_{3} \bar{q}_{2}, \quad c_{4}^{*}=c_{4}+c_{6} \bar{q}_{2}, \\
c_{5}^{*}=c_{5}+3 c_{7} \bar{q}_{2}, \quad h_{1}=3 c_{2} \bar{q}_{1}, \quad h_{2}=c_{3} \bar{q}_{1}, \quad h_{3}=2 c_{6} \bar{q}_{1} .
\end{gathered}
$$

It emerges that the static forces $f_{\text {ri }}$ modify the equilibrium configuration of the cable and, consequently, its elastic properties. In particular, the tangent stiffness matrix is no longer diagonal; therefore small-amplitude in-plane and out-of-plane oscillations are coupled and the linear frequencies depend on $\mu$. In addition, nonlinear $c^{*}$ coefficients are altered by the static forces and some new $h$-terms appear in the equations of motion.

\section{AMPLITUDE EQUATIONS}

The multiple-scales perturbation method is employed to obtain equations in amplitudes and phases. As a first step, a dimensionless small parameter $\varepsilon$ is introduced. It is assumed that the amplitude of motion and the damping are small and of the same order $\varepsilon$ :

$$
v_{i}=\varepsilon \hat{v}_{i}, \quad b_{j}=\varepsilon \hat{b}_{j}, \quad i=1,2 ; j=1, \ldots, 4 .
$$

In addition, two different hypotheses are made about the order of magnitude of the static displacements:

$$
\text { (a) } \bar{q}_{i}=O\left(v_{i}\right), \quad \text { (b) } \bar{q}_{i}>O\left(v_{i}\right) \text {. }
$$

In case (a) it is assumed that, due to the static forces, the cable configuration undergoes small deviations from the rest position, of the same order of the dynamic displacements, so that linear coupling ( $k_{2}$ - and $k_{3}$-terms) is weak. In case (b) it is assumed that the static displacements are larger than the dynamic components, so that linear coupling is strong. Case (a) is obviously much easier to deal with; however, it is expected that one has to resort to case (b) to describe accurately the motion when $\mu$ is moderately large.

The quadratic terms in equations (11) enhance strong modal coupling when the frequency of the in-plane motion is about twice that of out-of-plane motion, i.e.,

$$
\omega_{2}=2 \omega_{1}+\varepsilon \sigma, \quad \sigma=O(1),
$$

$\sigma$ being a detuning parameter. In this case, an internal resonance condition of type $1: 2$ occurs. This condition is verified around the first "cross-over" point of the cable [9], for which $\omega_{1}=1$ and $\omega_{2}=2$. Two slow time scales, $t_{1}$ and $t_{2}$, are introduced in addition to the fast scale $t_{0}$ :

$$
t_{n}=\varepsilon^{n} t, \quad n=0,1,2 .
$$


The two cases of equations (14) are separately dealt with in the sequel.

\subsection{SMALL STATIC DISPLACEMENTS}

By letting $\bar{q}_{i}=\varepsilon \hat{q}_{i}$, using equations (13) and omitting the hat, the equations of motion (11) can be expressed as

$$
\begin{gathered}
\ddot{v}_{1}+\omega_{1}^{2} v_{1}+\varepsilon\left(k_{1}^{\prime} v_{1}+k_{2}^{\prime} v_{2}+b_{1} \dot{v}_{1}+b_{2} \dot{v}_{2}+c_{1} v_{1} v_{2}+b_{5} \dot{v}_{1}^{2}+b_{6} \dot{v}_{2}^{2}+b_{7} \dot{v}_{1} \dot{v}_{2}\right) \\
+\varepsilon^{2}\left(k_{1}^{\prime \prime} v_{1}+k_{2}^{\prime \prime} v_{2}+c_{1}^{\prime} v_{1} v_{2}+h_{1} v_{1}^{2}+h_{2} v_{2}^{2}+c_{2} v_{1}^{3}+c_{3} v_{1} v_{2}^{2}+b_{8} \dot{v}_{2}^{3}\right)=0, \\
\ddot{v}_{2}+\omega_{2}^{2} v_{2}+\varepsilon\left(k_{3}^{\prime} v_{1}+k_{4}^{\prime} v_{2}+b_{3} \dot{v}_{1}+b_{4} \dot{v}_{2}+c_{4} v_{1}^{2}+c_{5} v_{2}^{2}+b_{9} \dot{v}_{1}^{2}+b_{10} \dot{v}_{2}^{2}+b_{11} \dot{v}_{1} \dot{v}_{2}\right) \\
+\varepsilon^{2}\left(k_{3}^{\prime \prime} v_{1}+k_{4}^{\prime \prime} v_{2}+c_{4}^{\prime} v_{1}^{2}+c_{5}^{\prime} v_{2}^{2}+h_{3} v_{1} v_{2}+c_{6} v_{1}^{2} v_{2}+c_{7} v_{2}^{3}+b_{12} \dot{v}_{2}^{3}\right)=0,
\end{gathered}
$$

where $k_{i}^{\prime}, c_{j}^{\prime}$ and $k_{i}^{\prime \prime}$ denote linear and quadratic parts in $\bar{q}_{k}$ of coefficients (12), respectively.

Displacements $v_{k}$ are expanded in $\varepsilon$-series and use is made of the time scales (16):

$$
v_{k}(t ; \varepsilon)=v_{k 0}\left(t_{0}, t_{1}, t_{2}\right)+\varepsilon v_{k 1}\left(t_{0}, t_{1}, t_{2}\right)+\varepsilon^{2} v_{k 2}\left(t_{0}, t_{1}, t_{2}\right)+\cdots, \quad k=1,2 .
$$

Equating coefficients of the same powers of $\varepsilon$, one obtains the perturbative equations [12], not shown here for the sake of brevity. At order one, the generating solution is:

$$
v_{k 0}\left(t_{0}, t_{1}, t_{2}\right)=A_{k}\left(t_{1}, t_{2}\right) \mathrm{e}^{i \omega_{k} t_{0}}+\bar{A}_{k}\left(t_{1}, t_{2}\right) \mathrm{e}^{-\mathrm{i} \omega_{k} t_{0}}, \quad k=1,2,
$$

where $A_{k}$ are complex functions of the slow scales and the overbar denotes the complex conjugates. Elimination of the secular terms at the higher levels of approximation ( $\varepsilon$ and $\varepsilon^{2}$ orders) leads to conditions of the type

$$
\begin{aligned}
\mathrm{d}_{1} A_{1}=\mathfrak{I}_{11}\left(A_{1}, \bar{A}_{1} A_{2} ; \mu\right), & \mathrm{d}_{2} A_{1}=\mathfrak{I}_{21}\left(A_{1}, \bar{A}_{1} A_{2}, A_{1} A_{2} \bar{A}_{2}, A_{1}^{2} \bar{A}_{1} ; \mu\right), \\
\mathrm{d}_{1} A_{2}=\mathfrak{J}_{12}\left(A_{2}, A_{1}^{2} ; \mu\right), & \mathrm{d}_{2} A_{2}=\mathfrak{I}_{22}\left(A_{2}, A_{1}^{2}, A_{1} \bar{A}_{1} A_{2}, A_{2}^{2} \bar{A}_{2} ; \mu\right),
\end{aligned}
$$

where $\mathrm{d}_{n}=\partial / \partial t_{n}$. Equations (20) govern the amplitude modulation on the slow scales $t_{1}$ and $t_{2}$. It is possible to go back to the true time $t$ by combining them, noting that $\mathrm{d} / \mathrm{d} t=\mathrm{d}_{0}+\varepsilon \mathrm{d}_{1}+\varepsilon^{2} \mathrm{~d}_{2}$ and the $A_{\mathrm{k}} \mathrm{s}$ are independent of $t_{0}$. Upon re-absorbing parameter $\varepsilon$, the following amplitude equations are obtained,

$$
\dot{A}_{1}=\mathfrak{J}_{1}\left(A_{1}, \bar{A}_{1} A_{2}, A_{1} A_{2} \bar{A}_{2}, A_{1}^{2} \bar{A}_{1} ; \mu\right), \quad \dot{A}_{2}=\mathfrak{I}_{2}\left(A_{2}, A_{1}^{2}, A_{1} \bar{A}_{1} A_{2}, A_{2}^{2} \bar{A}_{2} ; \mu\right),
$$

where $\mathfrak{I}_{k}=\mathfrak{I}_{1 k}+\mathfrak{J}_{2 k}$. By introducing the polar form

$$
A_{k}=\frac{1}{2} a_{k} \mathrm{e}^{\mathrm{i} \theta_{k}}, \quad k=1,2,
$$

where $a_{k}$ and $\vartheta_{k}$ are the amplitude and phase of $A_{k}$, respectively, and separating equations (21) into real and imaginary parts, four state equations in the real variables $a_{k}$ and $\vartheta_{k}$ are obtained. These equations can be transformed into an autonomous system by letting

$$
\gamma=\vartheta_{2}-2 \vartheta_{1}+\sigma t
$$

The result is the set of three equations

$$
\begin{gathered}
\dot{a}_{1}=a_{1}\left[p_{1}+p_{2} a_{2} \sin \gamma+p_{3} a_{2} \cos \gamma\right], \\
\dot{a}_{2}=p_{4} a_{2}+p_{5} a_{2}^{3}+p_{6} a_{1}^{2} \sin \gamma+p_{7} a_{1}^{2} \cos \gamma, \\
a_{1} a_{2} \dot{\gamma}=a_{1}\left[p_{8} a_{2}+p_{9} a_{2}^{3}+p_{10} a_{1}^{2} a_{2}-p_{7} a_{1}^{2} \sin \gamma+p_{6} a_{1}^{2} \cos \gamma-2 p_{3} a_{2}^{2} \sin \gamma+2 p_{2} a_{2}^{2} \cos \gamma\right],
\end{gathered}
$$

where the coefficients $p_{i}$ are generally functions of the non-dimensional mean wind speed $\mu$; their expressions are given in Appendix B. 
Equations (24) are formally identical to those obtained in reference [7], where a slightly different procedure was followed, based on a perturbative approximation of the static equilibrium path. They represent a non-linear dynamic system of state variables $\left(a_{1}, a_{2}, \gamma\right)$ asymptotically equivalent to the original system (11).

\subsection{LARGE STATIC DISPLACEMENTS}

By using the ordering (13) in the equations of motion (11) and assuming $\bar{q}_{i}=O(1)$, non-linear equations still coupled in the linear part are obtained. To make their treatment simpler, it is convenient to perform a linear transformation to uncouple the linear part. By setting $\{\mathbf{v}\}=[U]\{\xi\}$, where

$$
[U]=\left[\begin{array}{cc}
1 & \alpha_{2} \\
\alpha_{1} & 1
\end{array}\right]
$$

is the $2 \times 2$ modal matrix and $\{\xi\}$ the vector of the principal co-ordinates, substituting in equations (11) and pre-multiplying them by $[U]^{-1}$, the following equations are obtained:

$$
\begin{aligned}
\xi_{1}+ & \dot{\omega}_{1}^{2} \xi_{1}+\varepsilon\left(r_{1} \dot{\xi}_{1}+r_{2} \dot{\xi}_{2}+s_{1} \xi_{1}^{2}+s_{2} \xi_{1} \xi_{2}+s_{3} \xi_{2}^{2}+r_{5} \dot{\xi}_{1}^{2}+r_{6} \xi_{1} \dot{\xi}_{2}+r_{7} \dot{\xi}_{2}^{2}\right) \\
& +\varepsilon^{2}\left(s_{4} \xi_{1}^{3}+s_{5} \xi_{1}^{2} \xi_{2}+s_{6} \xi_{1} \xi_{2}^{2}+s_{7} \xi_{2}^{3}+r_{8} \dot{\xi}_{1}^{3}+r_{9} \dot{\xi}_{1}^{2} \dot{\xi}_{2}+r_{10} \dot{\xi}_{1} \dot{\xi}_{2}^{2}+r_{11} \xi_{2}^{3}\right)=0, \\
\xi_{2}+\tilde{\omega}_{2}^{2} \xi_{2} & +\varepsilon\left(r_{3} \xi_{1}+r_{4} \dot{\xi}_{2}+s_{8} \xi_{1}^{2}+s_{9} \xi_{1} \xi_{2}+s_{10} \xi_{2}^{2}+r_{12} \dot{\xi}_{1}^{2}+r_{13} \xi_{1} \xi_{2}+r_{14} \dot{\xi}_{2}^{2}\right) \\
+ & \varepsilon^{2}\left(s_{11} \xi_{1}^{3}+s_{12} \xi_{1}^{2} \xi_{2}+s_{13} \xi_{1} \xi_{2}^{2}+s_{14} \xi_{2}^{3}+r_{15} \dot{\xi}_{1}^{3}+r_{16} \dot{\xi}_{1}^{2} \dot{\xi}_{2}+r_{17} \dot{\xi}_{1} \xi_{2}^{2}+r_{18} \xi_{2}^{3}\right)=0 .
\end{aligned}
$$

Here the frequencies $\tilde{\omega}$ and the coefficients $r$ and $s$ are listed in Appendix C. By performing the same steps as in the previous section, equations of type (21) are obtained, though with different coefficients. In particular, some coefficients which are purely imaginary in equations (21) are instead complex in the present case. Therefore, some extra terms appear with respect to equations (24) when the polar form of the complex amplitudes is introduced. The resulting amplitude and phase modulation equations are

$$
\begin{gathered}
\dot{a}_{1}=a_{1}\left[p_{1}+p_{2} a_{2} \sin \gamma+p_{3} a_{2} \cos \gamma+p_{11} a_{1}^{2}+p_{12} a_{2}^{2}\right], \\
\dot{a}_{2}=p_{4} a_{2}+p_{5} a_{2}^{3}+p_{6} a_{1}^{2} \sin \gamma+p_{7} a_{1}^{2} \cos \gamma+p_{13} a_{1}^{2} a_{2}, \\
a_{1} a_{2} \gamma=a_{1}\left[p_{8} a_{2}+p_{9} a_{2}^{3}+p_{10} a_{1}^{2} a_{2}-p_{7} a_{1}^{2} \sin \gamma+p_{6} a_{1}^{2} \cos \gamma-2 p_{3} a_{2}^{2} \sin \gamma+2 p_{2} a_{2}^{2} \cos \gamma\right] .
\end{gathered}
$$

Coefficients $p_{1}-p_{10}$ are re-defined in Appendix C, together with the new $p_{11}-p_{13}$ coefficients. It should be remembered, however, that $a_{1}, a_{2}$ and $\gamma$ now represent the amplitudes and the phase difference of the principal coordinates $\xi_{i}$.

\section{AMPLITUDE EQUATIONS ANALYSIS}

\subsection{STEADY STATE SOLUTIONS}

The fixed points $\left(a_{1}, a_{2}, \gamma\right)$ of the dynamical systems (24) and (27) correspond to steady state solutions of the original system (11). 
The case of small static displacements is considered first. By truncating the expansion (18) at the $\varepsilon$-order, steady state solutions of equations (24) are found to be

$$
\begin{aligned}
v_{1}(t)= & a_{1} \cos \Omega_{1} t-\Lambda_{1} a_{2} \sin \left(\Omega_{2} t+\gamma\right)+\Lambda_{2} a_{2} \cos \left(\Omega_{2} t+\gamma\right)+\frac{1}{2} \Lambda_{3} a_{1}^{2} \cos 2 \Omega_{1} t \\
+ & \frac{1}{2} \Lambda_{4} a_{2}^{2} \cos \left(2 \Omega_{2} t+2 \gamma\right)+\frac{1}{2} \Lambda_{5} a_{1} a_{2} \cos \left[\left(\Omega_{1}+\Omega_{2}\right) t+\gamma\right]+\frac{1}{2} \Lambda_{6} a_{1}^{2}+\frac{1}{2} \Lambda_{7} a_{2}^{2}, \\
v_{2}(t)= & a_{2} \cos \left(\Omega_{2} t+\gamma\right)-\Lambda_{8} a_{1} \sin \Omega_{1} t+\Lambda_{9} a_{1} \cos \Omega_{1} t+\frac{1}{2} \Lambda_{10} a_{2}^{2} \cos \left(2 \Omega_{2} t+2 \gamma\right) \\
& +\frac{1}{2} \Lambda_{11} a_{1} a_{2} \cos \left[\left(\Omega_{2}-\Omega_{1}\right) t+\gamma\right]+\frac{1}{2} \Lambda_{12} a_{1} a_{2} \cos \left[\left(\Omega_{1}+\Omega_{2}\right) t+\gamma\right] \\
& +\frac{1}{2} \Lambda_{13} a_{1}^{2}+\frac{1}{2} \Lambda_{14} a_{2}^{2} .
\end{aligned}
$$

Here an arbitrary initial phase has been set equal to zero. In equations (28)

$$
\Omega_{1}=\Omega_{2} / 2, \quad \Omega_{2}=\omega_{2}+p_{8}^{\prime}-p_{7}\left(a_{1}^{2} / a_{2}\right) \sin \gamma+p_{6}\left(a_{1}^{2} / a_{2}\right) \cos \gamma+p_{10}^{\prime} a_{1}^{2}+p_{9}^{\prime} a_{2}^{2} .
$$

are the amplitude-dependent frequencies of the periodic motion (non-linear frequencies). Coefficients $p_{i}$ and $\Lambda_{i}$ are given in Appendix B. In equation (29b) $p_{8}^{\prime}$ represents the correction of the linear undamped frequency $\omega_{2}$ due to the damping terms $b_{1}, \ldots b_{4}$. Equations (29) contain, as a particular case (no aerodynamic terms or damping), the quadratic approximation of the non-linear frequency $\Omega_{2}$ of undamped free planar motions given in ref rence [4]. It should be noted that, at the leading order, the in-plane $v_{2}$-component oscillates at a frequency double that of the out-of-plane $v_{1}$-component, with a phase-difference $\gamma$.

When large static displacements are considered, equations (28) and (29) still hold, with the out-of-plane and in-plane displacements $v_{1}(t)$ and $v_{2}(t)$ replaced by the principal co-ordinates $\xi_{1}(t)$ and $\xi_{2}(t)$, respectively, the natural frequencies $\omega_{i}$ substituted by the modified frequencies $\tilde{\omega}_{i}$, and with the coefficients $p_{i}$ and $\Lambda_{i}$ defined in Appendix C. Since $v_{1}(t)=\xi_{1}(t)+\alpha_{2} \xi_{2}(t)$ and $v_{2}(t)=\alpha_{1} \xi_{1}(t)+\xi_{2}(t)$ according to equation (25), each component $v_{i}(t)$ oscillates at the leading order with two frequencies in 1:2 ratio.

\subsection{BIFURCATION AND STABILITY ANALYSIS}

From the analysis of equations (24) and (27) the existence of the following branches of fixed points is proved (see Figures 3(a, b)): branch I, $a_{1}=a_{2}=0, \gamma$ arbitrary, $\forall \mu$; branch II, $a_{1}=0, a_{2}=a_{2}(\mu)=\sqrt{-p_{4}(\mu) / p_{5}(\mu)}, \gamma$ arbitrary; branches III and IV, $a_{1}=a_{1}(\mu)$, $a_{2}=a_{2}(\mu), \gamma=\gamma(\mu)$.

First, the bifurcation of branch I is studied. When $a_{1} \rightarrow 0, \mathrm{a}_{2} \rightarrow 0, \mu \rightarrow \mu_{c}$, equations (24) and (27) reduce to

$$
a_{1} p_{i}^{c}=0, \quad a_{2} p_{4}^{c}=0, \quad a_{1} a_{2} p_{8}^{c}=0,
$$

where $p_{i}^{c}=p_{i}\left(\mu_{c}\right)$. Two different solutions of equations (30) exist: $a_{2}$-bifurcation, $a_{1}=0$, $a_{2} \neq 0$ when $p_{4}^{c}=0 ; a_{1}$-bifurcation, $a_{1} \neq 0, a_{2}=0$ when $p_{1}^{c}=0$. In the first case branch II bifurcates in the $a_{2}$-direction (see Figures $3(\mathrm{a}, \mathrm{b})$ ) while, in the second case, branch IV bifurcates in the $a_{1}$-direction (see Figure 3(b)). In both cases galloping is monomodal, since only one mode is triggered at the bifurcation. However, in the postcritical range, while the motion remains monomodal along branch II, it becomes bimodal along branch IV.

The bifurcations of branch I and the pattern of branch II strongly depend on the magnitude of the static displacements. In fact, if static displacements are assumed small, $p_{1}<0$ for any $\mu$, since the drag coefficient $c_{d}$ is positive for any cross-section shape; therefore only in-plane $a_{2}$-bifurcations can occur. In addition, since $p_{4}(\mu)$ is a monotonic function of $\mu$ and $p_{5}(\mu)<0$, branch II intersects branch I only at the $\mathrm{B}_{1}$-point. Therefore, branch II is an open curve, similar to a typical one-d.o.f. vertical galloping diagram (see 


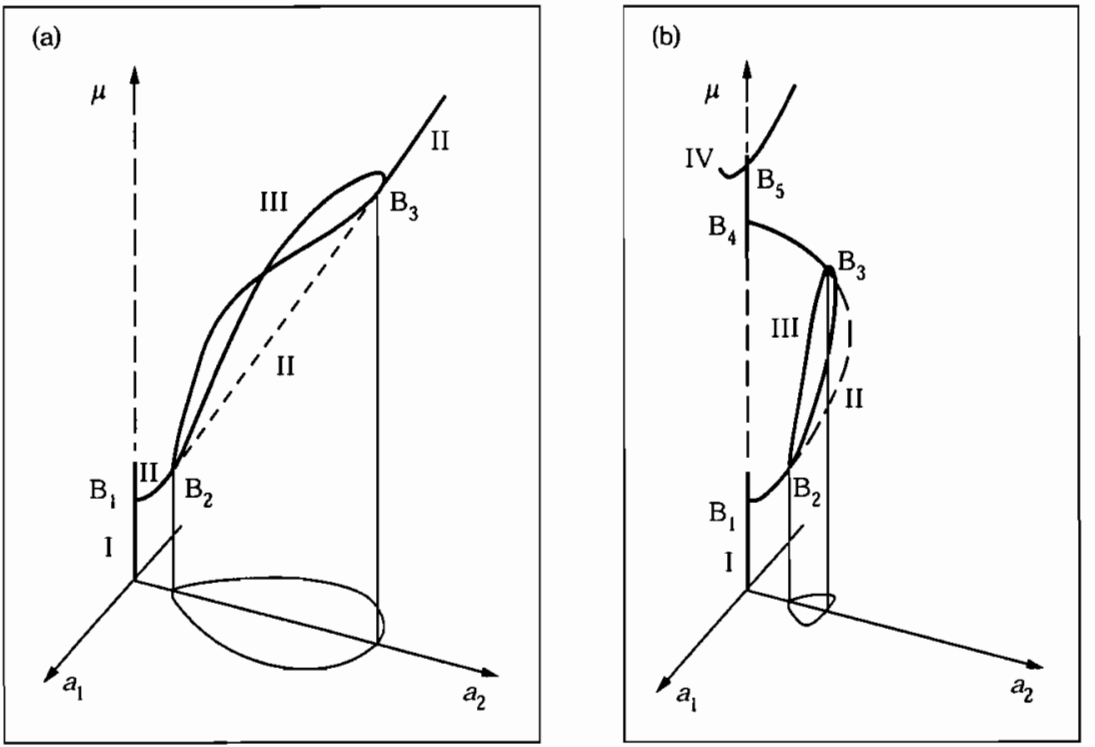

Figure 3. Steady state amplitude solutions vs. nondimensional wind velocity; (a) small and (b) large static displacement solutions; $(-)$ stable, $(---)$ unstable.

Figure 3(a)), though in the problem analyzed here, the motion is not purely planar, as can be seen from equations (28). On the contrary, if static displacements are assumed large, both kinds of bifurcation take place. In addition, $p_{4}(\mu)$ is a non-monotonic function of $\mu$, with $p_{5}(\mu)$ still negative; therefore branch II intersects branch I at $\mathrm{B}_{1}$ - and $\mathrm{B}_{4}$-points and disappears for higher $\mu$ 's: i.e., it is a closed curve (see Figure 3(b)). Since $p_{4}(\mu)$ represents the real part of an eigenvalue of the linearized system, it follows that branch II exists only for values of $\mu$ for which branch I is unstable.

The conclusion is that the small static displacement approximation is quite inadequate to correctly depict the qualitative scenario, if sufficiently large values of $\mu$ have to be considered. However, if the critical wind velocity is sufficiently low, it allows an accurate description of the first bifurcation and the initial postcritical behaviour. In particular, it furnishes a critical value $\mu_{c}$ which differs from the one drawn by the well-known Den Hartog criterion [1], since it accounts for the effects of the mean wind force in binormal direction $f_{10}$.

Next the bifurcation of branch II is analyzed. To this end one looks for the existence of two-component solutions $\left(a_{1} \neq 0, a_{2} \neq 0\right)$ close to the branch $\left(a_{1}, a_{2}\right)=\left(0, a_{2}(\mu)\right)$. By resolving the limit of equations (27) for $a_{1} \rightarrow 0, \gamma \rightarrow \gamma_{0}, \mu \rightarrow \mu_{0}, a_{2} \rightarrow a_{20}$, with $a_{20}=a_{2}\left(\mu_{0}\right)$, and by letting $p_{i}^{0}=p_{i}\left(\mu_{0}\right)$, the following equations are obtained:

$$
\begin{gathered}
p_{1}^{0}+p_{2}^{0} a_{20} \sin \gamma_{0}+p_{3}^{0} a_{20} \cos \gamma_{0}+p_{12}^{0} a_{20}^{2}=0, \\
p_{8}^{0} a_{20}+p_{9}^{0} a_{20}^{3}-2 p_{3}^{0} a_{20}^{2} \sin \gamma_{0}+2 p_{2}^{0} a_{20}^{2} \cos \gamma_{0}=0
\end{gathered}
$$

since the second of equations (27) gives an identity to the leading order. Equations (31) are a non-linear algebraic system with two unknowns, $\gamma_{0}$ and $\mu_{0}$. Each solution of this system determines a bifurcation point on the branch II, from which a branch III originates $\left(B_{2^{-}}\right.$and $B_{3}$-points in Figure $\left.3(\mathrm{a}, \mathrm{b})\right)$. It will be shown in the sequel that by using numerical solutions, branch III is a closed path, both in the small and large static displacements regime. Bimodal galloping occurs along the path as a consequence of 
the non-linear interaction between the two internally resonant modes, mainly governed by quadratic mechanical terms.

The stability of branch II cannot be studied by standard methods if the polar form (24) or (27) of the amplitude equations is considered, but use has to be made of the Cartesian form (see, e.g. reference [5]). However, stability has been checked here numerically by analyzing the variational equations of the equations of motion (11), based on the periodic solution (28), via the Floquet theory. It has been found that branch II is unstable between the bifurcation points $B_{2}$ and $B_{3}$, and stable elsewhere. $A$ very good agreement about the position of the bifurcation points in the two different approaches has been found. Finally, the stability of branch III has been analyzed via standard eigenvalue analysis. It has been found that it sometimes undergoes Hopf bifurcations, as illustrated below.

\section{NUMERICAL RESULTS AND DISCUSSION}

Some different aspects of the problem have been investigated in order to describe the mechanical behaviour of the cable. First, the aerodynamic cable properties are discussed in section 6.1; then, small oscillations around the static configuration are analyzed in section 6.2. A comparison between the previously developed perturbation solutions and a numerical integration of the equations of motion is presented in section 6.3. The influence of the cable's parameters on postcritical equilibrium patterns is analyzed in section 6.4 . Simplified models, in which only the most important aerodynamic terms are retained, are discussed in section 6.5. Transient and steady state motions are analyzed in section 6.6. All the results are obtained for a section stressed by a symmetric mean wind; the effects of non-symmetric wind pressures are studied in section 6.7 .

\subsection{AERODYNAMIC PROPERTIES}

Two cases have been considered with respect to wind flow: symmetric cross-sections stressed by the mean wind speed along the symmetry axis, and symmetric or non-symmetric cross-sections stressed in a generic direction. The first particular case involves simplifications both in the equations of motion and in the amplitude equations; the latter generic case is described by the full form of the equations.

In the case of symmetric flow, the cross-section characteristic and the aerodynamic properties are deduced from reference [3] for a U-shaped conductor, by assuming the section to be symmetrical with its maximum ice eccentricity facing the wind. The cable has a diameter of $2.81 \mathrm{~cm}$, a mass of $1.80 \mathrm{~kg} / \mathrm{m}$ (ice included), damping coefficients equal to $0.44 \%$ and axial rigidity of $29.7 \times 10^{6} \mathrm{~N}$. In the case of non-symmetric flow, the aerodynamic properties are deduced from reference [17], by taking a U-shaped section again now stressed by a rotated flow of about $44^{\circ}$ with respect to the symmetry axis. The section has the same characteristics as the symmetric case but greater ice thickness (wet snow with a maximum thickness of $12.6 \mathrm{~mm}$ ), for which different aerodynamic coefficients have been determined. In this latter case the mass is assumed to be $2.00 \mathrm{~kg} / \mathrm{m}$, ice included. For both cases the first in-plane and out-of-plane symmetric modes are considered.

In the case of flow to a symmetry axis, which is quite a widespread assumption in the classical examples of literature [18], one obtains considerable simplifications. Since the drag coefficient $c_{d}\left(\gamma_{a}\right)$ is a symmetric function and the lift coefficient $c_{l}\left(\gamma_{a}\right)$ a skew-symmetric function of the fluctuating angle of attack $\gamma_{a}$, the along-wind $c_{F 1}$ and transversal $c_{F 2}$ force coefficients are, respectively, symmetric and skew-symmetric as regards $\gamma_{a}$. For this reason, the aerodynamic forces $f_{i}$ have simplified expressions and, in the equations of motion (8), some coefficients vanish. In particular, there is a decoupling among the linear terms $\left(b_{2}=b_{3}=0\right)$ (i.e., proportional damping) and a lack of some non-linear terms 

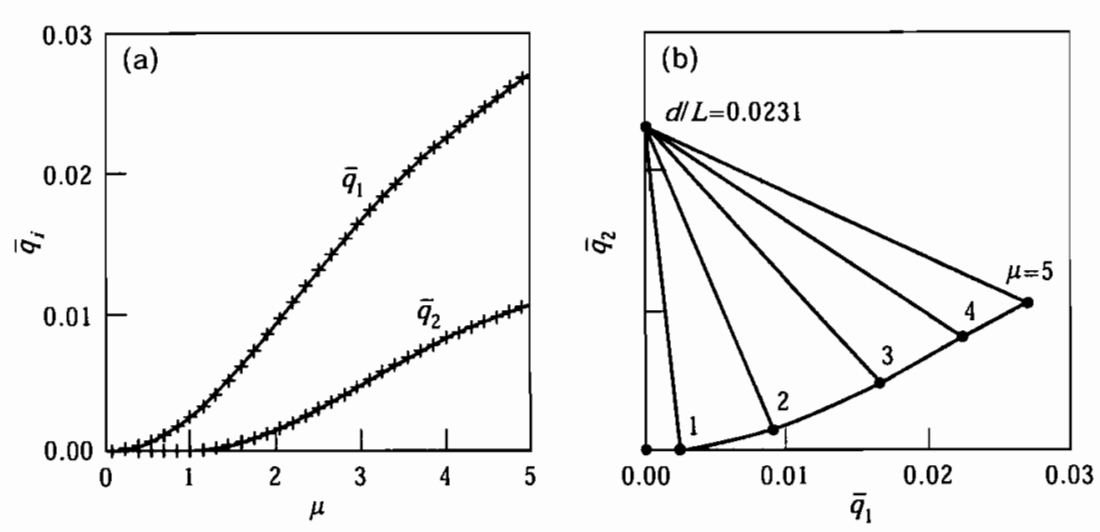

Figure 4. Static displacements of the mid-point of the cable; (a) displacements vs. wind velocity; (b) equilibrium path in the configuration plane.

( $b_{7}=b_{8}=b_{9}=b_{10}=0$ ), whereas some coefficients $p_{i}$ have simplified expressions. In particular, in the small static displacement case, $p_{1}=-b_{1} / 2$ and $p_{4}=-b_{4} / 2$ hold; therefore, the first bifurcation value $\mu_{c}$ satisfies the classical Den Hartog criterion, $b_{4}\left(\mu_{c}\right)=0$.

\subsection{SMALL OSCILLATIONS AROUND THE STEADY STATE CONFIGURATION}

As a sample case, a cable with dimensionless mechanical parameters $\alpha=1165, \beta=0.185$ is considered [11], hereafter called the basic example. According to Irvine's parameter [9] $\lambda^{2}=\alpha \beta^{2}=39.87213 \cong 4 \pi^{2}$, the cable is close to the first cross-over point $\left(\omega_{1}=1, \omega_{2}=2.00663\right)$. The steady state solution is determined as a function of the wind mean speed $\mu$ by numerically solving the system of non-linear equations (9) through Newton-Raphson techniques. The equilibrium points are subsequently approximated by using least squares in such a way as to obtain an analytical polynomial expression for the non-trivial equilibrium path $\bar{q}_{i}=\bar{q}_{i}(\mu)$. In Figure 4(a) the numerical solution and the polynomial regression (limited to sixth order) are compared; it appears that they are in excellent agreement. This numerical procedure has been preferred to a perturbative extrapolation of the static equilibrium path from the origin in order not to introduce further errors into the description of the cable non-linear dynamic response. In Figure 4(b) the static displacements of the mid-point of the cable are plotted for increasing wind velocities; straight lines represent the deformed sag of the cable. It is seen that, for high wind mean speed ( $\mu=4$ corresponds to about $40 \mathrm{~m} / \mathrm{s}$ ) the influence of steady state forces is remarkable; in particular the out-of-plane displacements are comparable to the initial in-plane sag.

Small oscillations around the steady-state configuration are then considered. Figure 5(a) shows the eigenvalues imaginary part of the linearized equation (11) versus $\mu$, obtained by solving numerically the characteristic equation. They are compared with order 1 and $\varepsilon^{2}$-order perturbative solutions, obtained in the two hypotheses of small (SSDS, dash-dotted lines) and large (LSDS, continuous lines) static displacements. The linear frequencies are strongly modified by the steady state forces when the mean wind speed is sufficiently high. Such behaviour is described by the SSDS only at the $\varepsilon^{2}$-order, since, in which the spectral properties of the generating system are not affected by steady state forces. On the contrary, the LSDS is very close to the numerical solution even at 
order 1 . As a consequence of the frequency modification, the detuning $\sigma$ rapidly increases and moves away from resonant conditions; therefore, the perturbative solution, which has been obtained for a one-to-two frequency ratio, loses precision for high wind speed.

Similar behaviour is found for the eigenvalues real part (see Figure 5(b)). The excellent approximation furnished by the LSDS allows one to describe correctly the $\mathrm{B}_{1}$ - and $\mathrm{B}_{4}$-bifurcations $\left(p_{4} \equiv \operatorname{Re} \lambda_{2}=0\right.$ ) and the $\mathrm{B}_{5}$-bifurcation $\left(p_{1} \equiv \operatorname{Re} \lambda_{1}=0\right.$ ) of branch I (see Figure 3(b)). In the basic example, the first bifurcation is also well approximated by the SSDS, corresponding to the Den Hartog criterion. However, particular cases could be encountered in which the stabilizing effect of the steady state forces on branch I influences the first bifurcation value and, in some borderline cases (i.e., large mechanical non-linearities or cross-sections not very sensitive to galloping), even disrupts this bifurcation.

Finally, Figure 5(c) shows the components $\alpha_{1}, \alpha_{2}$ of the eigenvectors (25). Their increasing values are a measure of the growing coupling between the in-plane and out-of-plane motions.

\subsection{NUMERICAL VALIDATION OF PERTURBATIVE SOLUTIONS}

With reference to the basic example, a quantitative comparison between the two perturbative solutions developed above is performed. In Figure 6 SSDS (thin lines) and
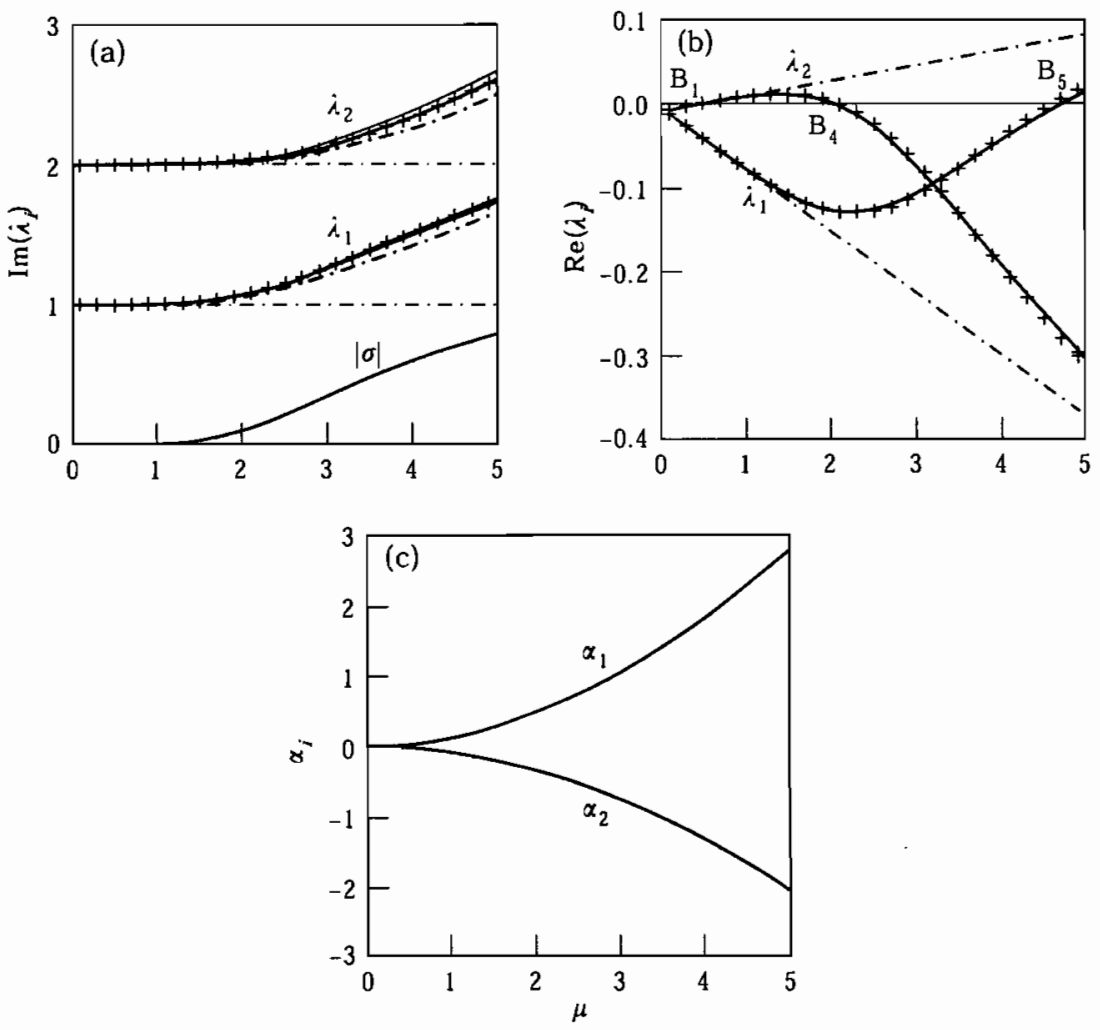

Figure 5. Eigensolutions of the linearized equations: (a) imaginary and (b) real part of the eigenvalues; (c) eigenvector components; thin lines: order 1 solution, thick lines: $\varepsilon^{2}$-order solution, + numerical solution. 

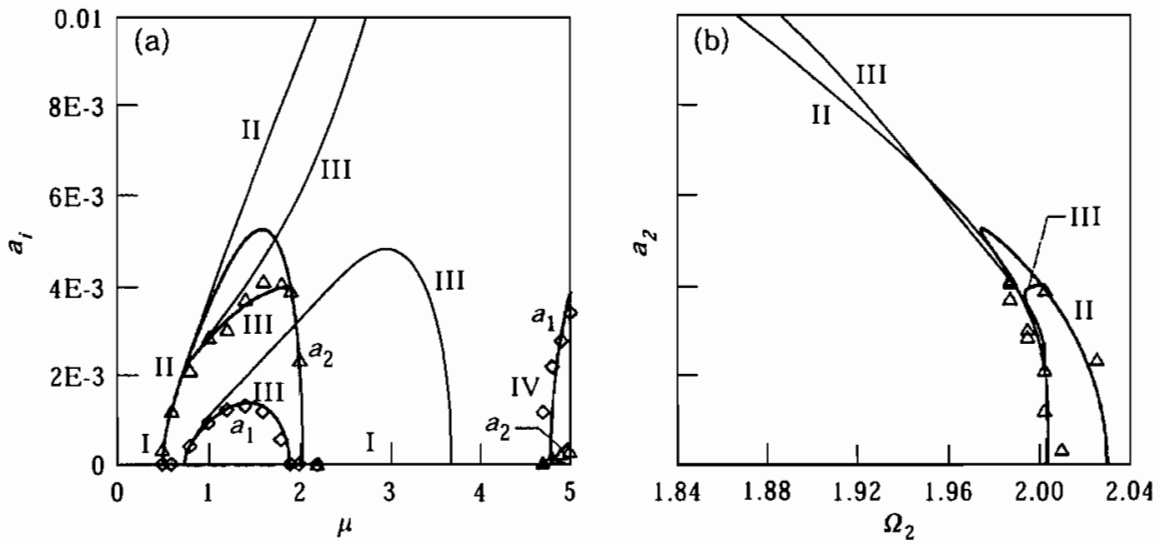

Figure 6. Comparison among SSDS (thin lines), LSDS (thick lines) and numerical solutions ( $\left.\diamond a_{1}, \triangle a_{2}\right):$ (a) postcritical branches; (b) non-linear frequencies.

LSDS (thick lines) are compared, both with regard to postcritical branches (Figure 6(a)) and non-linear frequencies (see Figure 6(b)). The postcritical branches in Figure 6(a) agree with the qualitative patterns of Figures 3(a,b). The monomodal branch II is an open curve in the SSDS and a closed curve in the LSDS. Branch III bifurcates from branch II in both solutions. Branch IV bifurcates from the trivial branch I only in the LSDS. Curves in Figure 6(b) represent the non-linear frequency $\Omega_{2}$ versus the in-plane amplitude $a_{2}$ (equation (29b)). The frequency curve II originates from the linear damped frequency, close to $\omega_{2}=2$. In the LSDS, $\Omega_{2}$ decreases until $a_{2}$ reaches a maximum (softening behaviour), then it increases until $a_{2}$ vanishes (hardening behaviour). In the SSDS, $\Omega_{2}$ always decreases. In both solutions, similarly to the amplitude curves, branch III bifurcates from branch II.
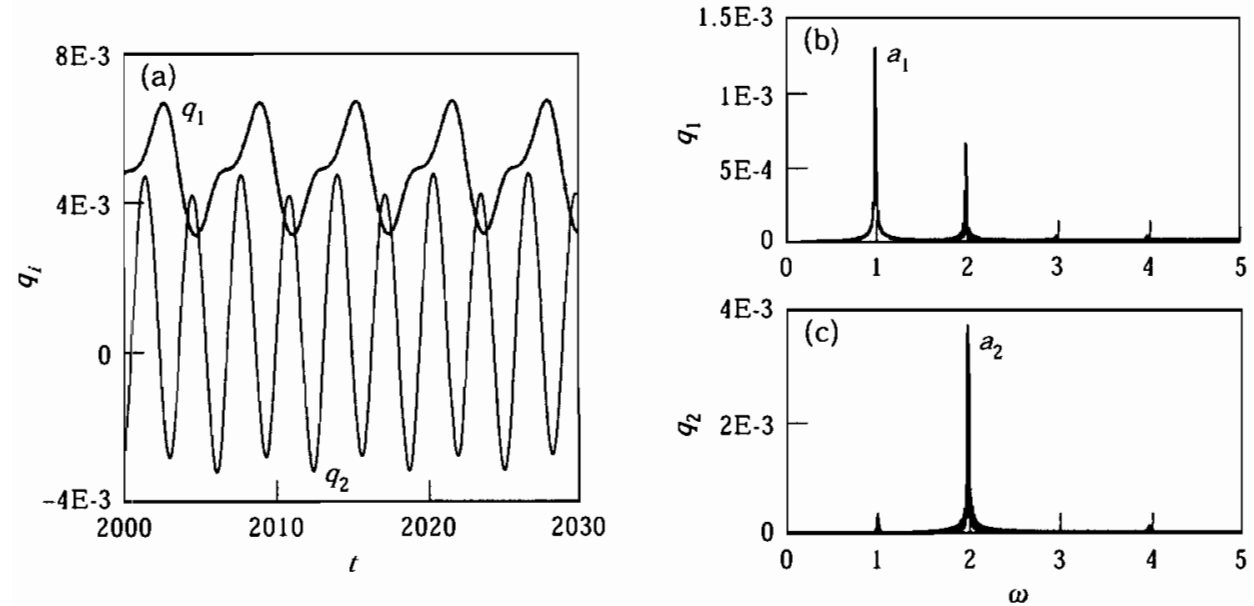

Figure 7. Numerical solutions for $\mu=1 \cdot 4$ : (a) steady state motions; (b) and (c) frequency spectra. 
Perturbative solutions are compared with numerical results (diamond points), obtained through direct integration of the Lagrangian equations (8). After specifying initial conditions, the integration is carried out until the system reaches a steady state (see Figure 7(a)), determined by checking the constancy of the peak-to-peak amplitudes over several consecutive periods. Steady state typically occurs at about $t=2000$. In order to identify the harmonic components of the steady motion, a fast Fourier transform (FFT) was performed over a sufficiently long interval of time with a zero mean signal (i.e., filtering the static component). The modal amplitudes $a_{1}$ and $a_{2}$ are approximately identified as the amplitude corresponding to the leading frequencies in $1: 2$ ratio (see Figures $7(\mathrm{~b}, \mathrm{c})$ ).

With regard to postcritical branches (see Figure 6(a)), it is noted that the LSDS is very close to the numerical solution and accurately describes the non-linear behaviour even at high wind speeds, though the errors grow due to the progressive detaching from resonant conditions. On non-linear frequencies (see Figure $6(\mathrm{~b})$, for $\mu \in(0,2-5)$ ) the errors of the LSDS appear to be larger. However, they are due to numerical problems related to the frequency discretization in FFT (in Figures 6 and $7 \Delta \omega=0.00767$ has been used). This circumstance highlights the benefit of the analytical solution as compared with the numerical solution.

In conclusion, the SSDS is able to fit the numerical solution only for low values of the non-dimensional wind speed $\mu$ while, for sufficiently high $\mu$, it fails to describe even the qualitative behaviour of the real branches. Therefore, reference is made in the sequel to LSDS. Previous results emphasize the importance of the steady state forcing terms. They are usually neglected in the literature, because they are considered as not influencing the bifurcation. In the problem dealt with here, however, they play an important role in the description of both the bifurcation and the postcritical behaviour.

\subsection{INFLUENCE OF CABLE PARAMETERS}

In order to study the influence of cable parameters on the equilibrium paths' shapes, three different iced suspended cables are considered with the same cross-section (i.e., same aerodynamic properties), different values of the dimensionless parameter $\beta$ $(0 \cdot 20,0 \cdot 185,0 \cdot 15)$ but the same values of the Irvine's parameter $\lambda^{2}=\alpha \beta^{2} \cong 4 \pi^{2}$. Therefore, the cables have the same detuning and dimensionless frequencies but different length and critical values of galloping. All the equilibrium branches and the non-linear frequencies of the three cables are drawn in Figure 8. From top to bottom $\beta$ decreases and $\alpha$ increases; therefore, since quadratic terms are proportional to $\alpha \beta$ and cubic terms to $\alpha$ (see Appendix A), all the geometric non-linearities increase. This circumstance has two effects. First, due to the major influence of the static displacements, the $\mathbf{B}_{4}$-bifurcation occurs at lower $\mu$-values (see Figure 5); consequently branches II and III exist in a smaller interval of $\mu$ and the static equilibrium configuration (branch I) regains stability in advance. Second, due to the major influence of the quadratic non-linearities, which are the most importantly responsible for the occurrence of coupled galloping, branch III exists in a wider range, if compared with the branch II domain. In summary, when geometrical non-linearities increase, the $B_{2}$ - and $B_{3}$-bifurcation points tend to the $B_{1}$ - and $B_{4}$-points, respectively.

The analysis performed above has permitted the highlighting of the occurrence on branch III of a new bifurcation phenomenon. When $\beta=0.15$ (see Figure 8(c)), at the $\mathrm{B}_{6}$ and $B_{7}$-points, a couple of eigenvalues of the variational matrix cross the imaginary axis; thus, a Hopf bifurcation occurs and a region of amplitude modulate motions arises between $B_{6}$ and $B_{7}$. An example is shown in Figure 9, obtained by numerically integrating the amplitude modulation equations (27) $\left(\mu=1.0\right.$ and initial conditions $\left.a_{1}=a_{2}=10^{-3}\right)$. There, the time-histories of the modal amplitudes (see Figure 9(a)) and the trajectory on 

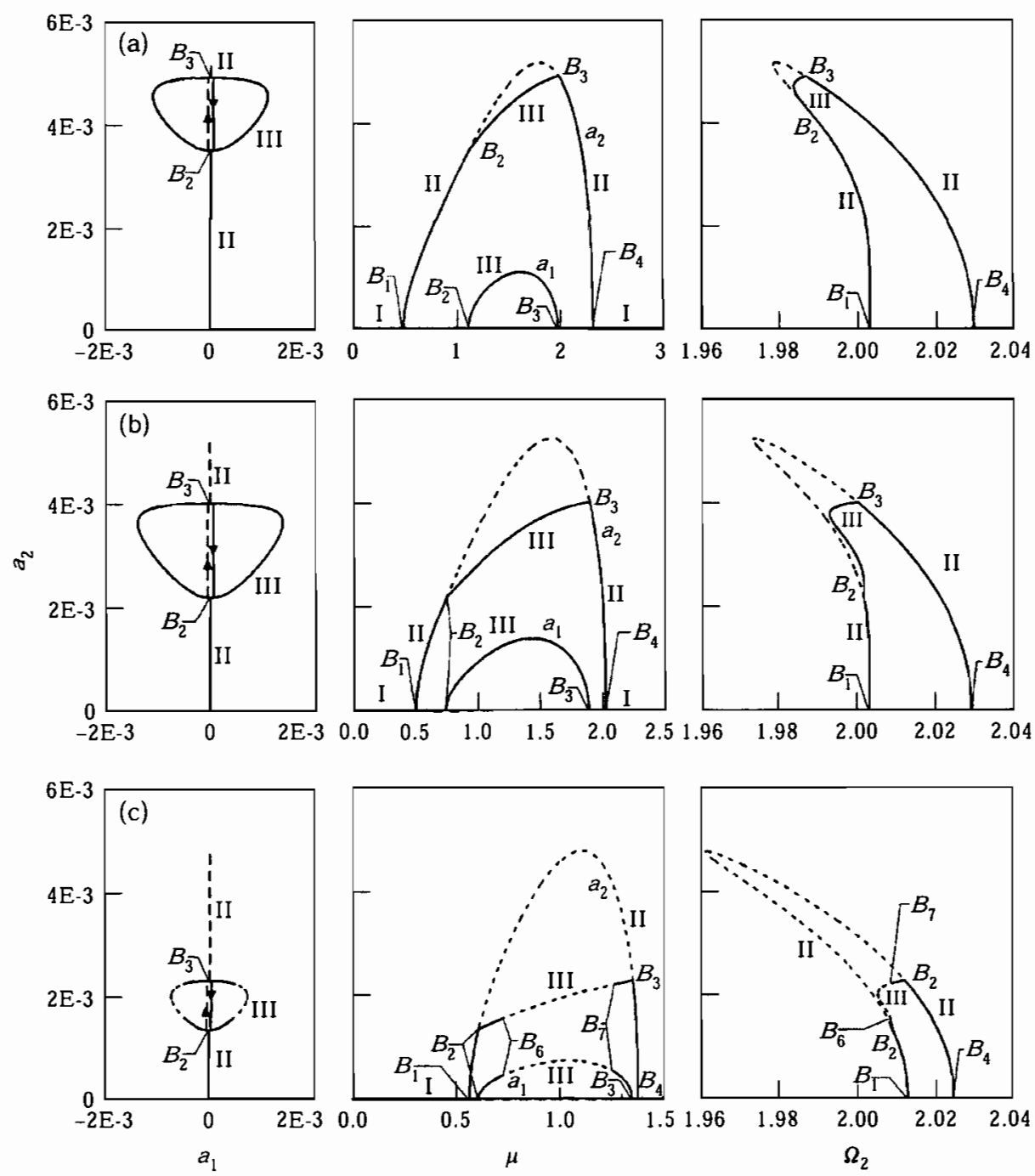

Figure 8. Non-linear equilibrium paths and in-plane frequencies for three different cables with constant parameter $\lambda^{2}$; (a) $\alpha=996.8031, \beta=0.20$; (b) $\alpha=1165, \beta=0.185$; (c) $\alpha=1772.0944, \beta=0.15$; $(\longrightarrow$ ) stable, (- --$)$ unstable.

the phase-plane (see Figure 9(b)) are plotted. The unstable equilibrium solution (diamond-point) is also drawn in Figure 9(b). It is seen that, after the transient has been exhausted, a limit-cycle is reached, along which the cable experiences a periodically amplitude-modulated motion. In the steady state regime, strong energy exchanges occur between in-plane and out-of-plane components, with a typical pulse behaviour. These results have also been confirmed by numerical integrations of the Lagrangian motion equations (8).

The robustness of previous results when the detuning $\sigma$ is varied is now investigated. In Figure 10 results relative to two different cables are illustrated. The cables have the same cross-section and geometric characteristics (in such a way non-dimensional amplitudes and 


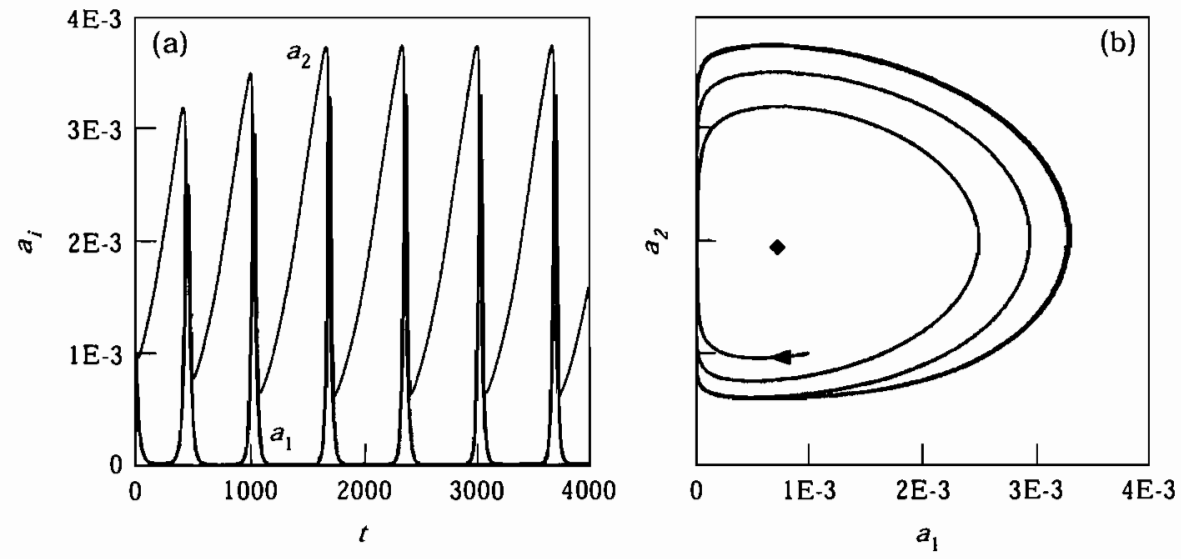

Figure 9. Amplitude modulate motions for $\mu=1 \cdot 0$ : (a) amplitude time-histories; (b) trajectory on the phase-plane ( $\$$ unstable equilibrium position; - limit cycle).

wind velocity are comparable), but different sags, for which $\sigma=-0 \cdot 10 \quad(\alpha=990$, $\beta=0.185$, thin lines) or $\sigma=0.19(\alpha=1272.83, \beta=0.2021$, thick lines). It is apparent that the bimodal galloping still holds in a frequency range around the first cross-over point, particularly wide for positive detuning, for which mechanical quadratic terms are larger.

\subsection{SIMPLIFIED MODELS}

It is worth discussing previous results through simplified models, drawn from the complete equations by neglecting some non-linear terms. The discussion will make it possible to understand the importance of several non-linear forces on both qualitative and quantitative system mechanical behaviour. The amplitude equation coefficients $p_{i}$ depend both on mechanical (coefficients $c_{i}$ ) and aerodynamic (coefficients $b_{i}$ ) non-linearities. However, from the numerical values they assume in the examples illustrated above, it
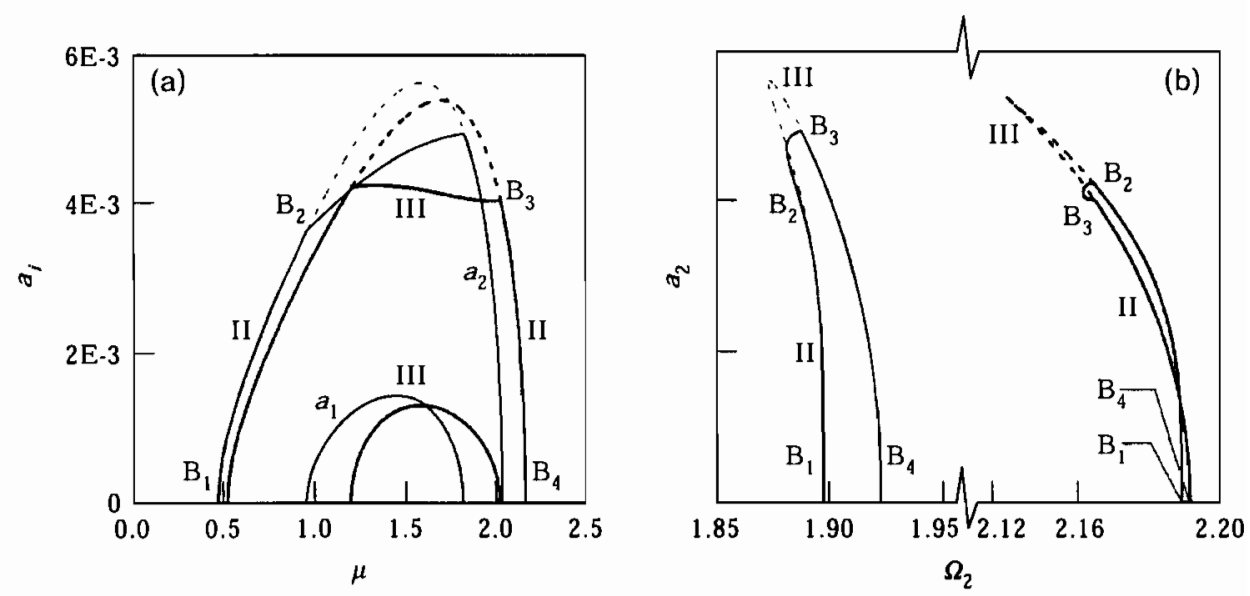

Figure 10. Detuning effects ( $\sigma=-0 \cdot 10$, thin lines; $\sigma=-0.19$, thick lines): (a) postcritical branches; (b) non-linear frequencies. 

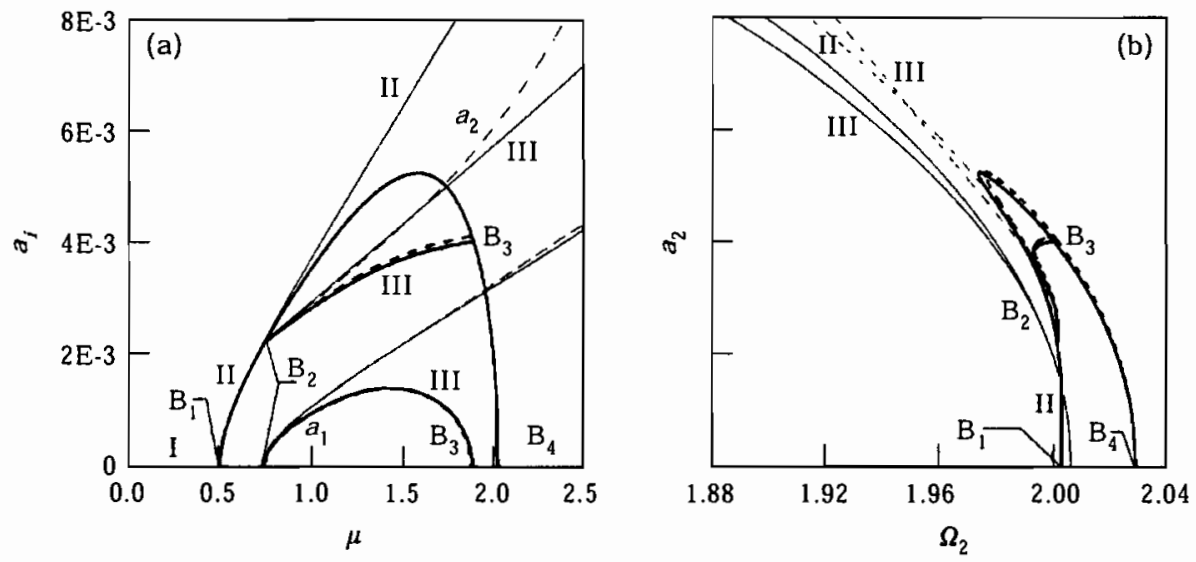

Figure 11. (a) Modal amplitudes and (b) in-plane non-linear frequencies vs. $a_{2}$; complete model: LSDS (continuous thick lines) and SSDS (dashed thin lines); LSDS simplified models: all aerodynamic non-linearities negiected, except for $b_{12}$, accounting for (dashed thick lines) or neglecting (continuous thin lines) steady state forcing terms.

appears that coefficients $p_{i}$ are weakly affected by non-linear aerodynamic terms, that slightly modify the mechanical ones, with the exception of the coefficients $p_{5}, p_{11}, p_{12}, p_{13}$, which depend only on the cubic aerodynamic non-linear terms $b_{8}$ and $b_{12}$. The latter, through the $p_{s}$ coefficient, plays a fundamental role in the description of branches II while $b_{8}$ is nil for symmetry. Neglecting all non-linear aerodynamic terms except $b_{12}$, one obtains the approximate (dashed thick lines) equilibrium paths shown in Figure 11(a). All the qualitative properties of the complete model are preserved; in fact, branch II is exactly reproduced and branch III is very well approximated.

Among the geometric non-linearities, the most important from a qualitative point of view appear to be the quadratic ones, responsible for the internal resonance phenomenon, as highlighted in many works on this subject (see, e.g., reference [5]). Therefore, the bifurcation point $B_{2}$ essentially depends on the quadratic geometric non-linear terms, in particular on resonant ones $c_{1}$ and $c_{4}$. If all non-linear geometric terms are neglected and only aerodynamic coefficients are retained, the small and large static displacement solutions coincide. Moreover a drastic simplification occurs, since several amplitudeequation coefficients vanish $\left(p_{2}=p_{3}=p_{6}=p_{7}=p_{11}=p_{12}=p_{13}=0\right)$. Then the study of the fixed points of equation $\left(27_{1}\right)$ reduces to $p_{1} a_{1}=0$; since $p_{1} \neq 0 \forall \mu, a_{1}$ is nil. Therefore two-component steady state motions (bimodal galloping) are lost if geometric non-linearities are not taken into account. Moreover, branch II is always an open stable curve and coincides with the classical one-d.o.f. galloping branch (see, e.g., reference [18]). These facts point out the importance of geometric non-linear terms in the qualitative description of branches.

If constant forces are omitted and only linear and non-linear $b_{12}$ terms retained, the branches plotted with continuous thin lines in Figure 11(a) are obtained. They are similar to the SSDS of Figure 6, here reported with dashed thin lines. It is seen that branch II does not close; moreover it regains stability only at very high wind velocity values.

The importance of steady state forcing terms clearly emerges also in the analysis of the in-plane non-linear frequency $\Omega_{2}$ on branch II, still referred to the basic case (see Figure 1l(b)). With respect to the classical one-d.o.f. galloping theory where the frequency is 
amplitude-independent (see, e.g., reference [18]), a frequency correction occurs here owing to geometrical and aerodynamic effects. The aerodynamic effects are mainly due to the cubic term $b_{12}$ that, although it does not enter directly in equations (29), governs the steady state amplitude $a_{2}$ and, consequently, the non-linear frequency. The simplified model accounting for steady state forces leads to numerical results practically coincident with the complete model, preserving all its properties. On the contrary, when steady-state forces are neglected, the strong change of non-linear frequency curvature and the successive $\mathbf{B}_{4}$-bifurcation are lost.

\subsection{TRANSIENT AND STEADY STATE MOTIONS}

Transient motions are analyzed through direct numerical integration of the amplitude equations (27). Reference is made to the basic example by assuming a non-dimensional wind speed $\mu$ in the range of existence of (stable) bimodal galloping $(\mu=1 \cdot 6)$. By perturbing the trivial equilibrium position, the motion illustrated in the $\left(a_{1}, a_{2}\right)$ phase-plane (see Figure 12(a)) and the associated amplitude time-histories (see Figure 12(b)) arise. It is seen that the trajectory is first rapidly attracted by the unstable branch II (represented by the higher ordinate point in Figure 12(a)); then the motion evolves towards the stable branch III where a steady state two-component oscillation takes place. During the transient, amplitudes larger than the regime amplitudes are reached.

The trajectories described by the cable mid-point $(s=1 / 2)$ are then analyzed. By using equations (10) and (28) (with $v_{i}$ replaced by $\left.\xi_{i}\right)$, the first-order approximation $\left(q_{i}=q_{n}\right.$, thin line) and the second-order approximation $\left(q_{i}=q_{i 0}+q_{i l}\right.$, thick line) to the periodic motion are illustrated in Figure 13(a). The non-trivial static equilibrium configuration is also represented by a cross-point in the figure. By comparing the two approximations, it appears that higher order terms slightly modify the trajectories while preserving their qualitative behaviour. Moreover, due to the presence of steady state forces, the out-of-plane displacements are always positive; therefore the cable never crosses the reference vertical configuration. In Figure 13(b) the second order approximation and the numerical results (diamond points) obtained through the direct integration of the
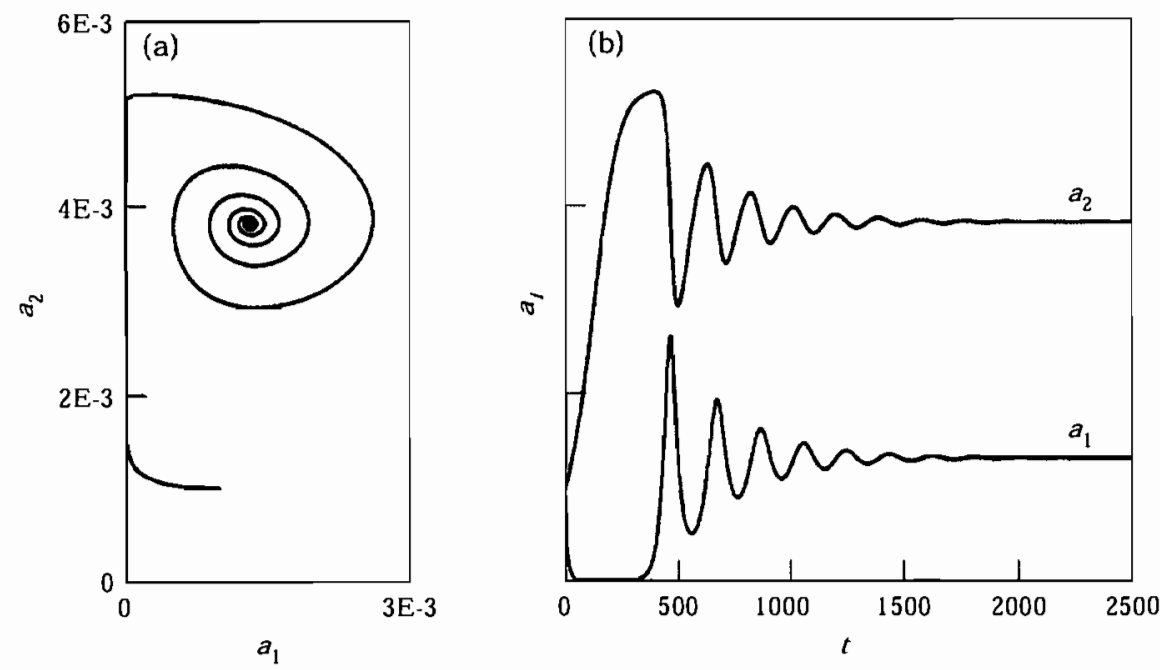

Figure 12. (a) Trajectories in the phase-plane and (b) amplitude time-histories for $\mu=1.6$. 


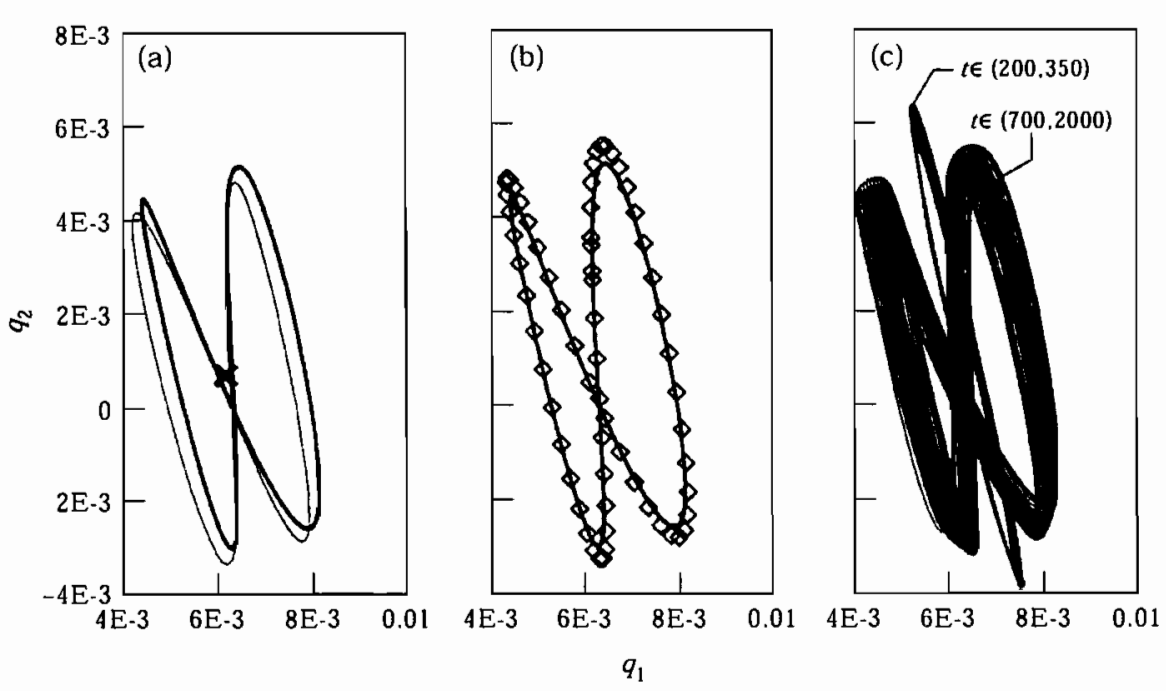

Figure 13. Motions of the cable at the mid-span for $\mu=1 \cdot 6$; (a), (b) steady state motion $\left(-\mathrm{q}_{n,},-q_{n}+q_{i 1}\right.$, $x$ static solution, $\diamond$ numerical solution); (c) transient motion.

Lagrangian equations (8) are compared. Excellent agreement is found since small errors occur only around the motion inversion points.

Transient motions of the cable at the mid-span are represented in Figure 13(c) by using equations (28) with $\left(a_{1}, a_{2}, \gamma\right)$ taken from the previous analysis (see Figure 12). The trajectory is initially close to an ellipse representing the unstable steady state motion on branch II; then it tends to the closed trajectory of Figure 13(b).

\subsection{NON-SYMMETRIC FLOW EFFECTS}

The effects of a non-symmetric flow are now analyzed. The previous sample cable is considered again, but its aerodynamic properties are modified according to section 6.1. First, the $\mathbf{B}_{\mathbf{1}}$-bifurcation on branch $\mathrm{I}$ is studied. The real part of the eigenvalues of the Jacobian matrix at the equilibrium position are plotted in Figure 14(a) versus the wind velocity. Both numerical (cross-points) and perturbative (LSDS continuous thick line, SSDS dashed thin line) results are shown. In addition the $b_{4}(\mu)$ linear function is also plotted, according to the Den Hartog criterion. It is seen that LSDS and numerical solutions are in excellent agreement, while SSDS diverges. However, the latter furnishes a very good approximation to the critical wind velocity value. On the contrary, if the Den Hartog criterion $b_{4}(\mu)=0$ is applied, i.e., if the coupling between the in-plane and out-of-plane displacements is ignored, an incorrect critical velocity, about $40 \%$ greater than the true value, is found. Therefore, different from the symmetric case, this simplistic criterion gives a dangerous non-protective estimate of the first bifurcation point. This circumstance can be explained by referring to the structure of the linear damping matrix in the modal basis. It is generally a full matrix (i.e., the damping is of non-proportional type), due to coupling effects produced both by static and aerodynamic interaction forces. However, the former are small at low wind velocity while the latter exist only in non-symmetric systems. Therefore, in the symmetric case the coupling is weak and the Den Hartog criterion is still quite accurate, while in the non-symmetric case the coupling is strong and the criterion is no longer valid. 

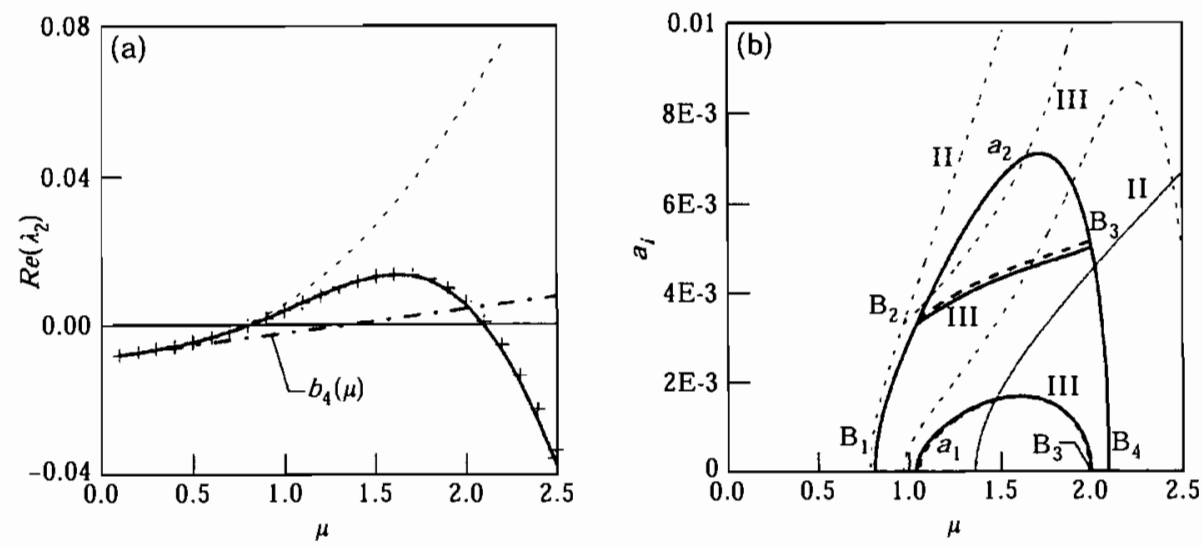

Figure 14. (a) Critical eigenvalue and (b) modal amplitudes for the basic example in non-symmetric flow; complete model: LSDS (continuous thick lines) and SSDS (dashed thin lines); LSDS simplified models: all aerodynamic non-linearities neglected, except for $b_{8}$ and $b_{12}$, accounting for (dashed thick lines) or neglecting (continuous thin lines) steady state forcing terms; + numerical solution.

Next the postcritical equilibrium branches are analyzed. In Figure 14(b) a comparison between the complete model (LSDS, continuous thick curves) and the simplified model (dashed thick curves), obtained by neglecting all the aerodynamic non-linearities except the cubic terms $b_{8}$ and $b_{12}$, is performed. It confirms previous conclusions about the really important forces (see section 6.5). The SSDS (dashed thin lines) is close to the previous solutions for sufficiently low $\mu$; in particular, an excellent approximation of the first bifurcation point and a good estimation of the second bifurcation point is found. However, if Figure 14(b) is compared with Figure 1 l(a), relative to the symmetric case, a not so good approximation emerges in the non-symmetric case. This occurrence is probably due to the major importance of the static forces, which in the non-symmetric problem act in both planes. Finally, if steady state forces are totally omitted (continuous thin lines) a strong alteration of the branches, also at low vibration amplitudes, is found.

\section{CONCLUSIONS}

In this paper, the non-linear response of a suspended cable in internal 1:2 resonance condition, excited by a transversal wind flow, has been analyzed by means of two different perturbative solutions, called small (SSDS) and large (LSDS) static displacements solutions, since they are based on a different ordering of the static displacements due to the steady-state wind forces. Both geometric and aerodynamic non-linearities have been considered by using consistent models. The approach presents some differences with respect both to non-linear cable dynamics literature, where the fluid-structure forces are often modelled in an inaccurate way, and to technical literature on galloping, where geometrical non-linearities are often ignored. The following conclusions can be drawn. (1) The SSDS is simpler but reliable only at low wind velocity $\mu$. The LSDS furnishes an excellent approximation of the cable's postcritical behaviour in a wide range of velocities, as confirmed by numerical integrations of the Lagrangian non-linear equations. (2) When the mean wind speed $\mu$ reaches a critical value, a (prevalently) in-plane oscillation 
(monomodal galloping) occurs; for increasing values of $\mu$, the monomodal oscillation becomes unstable and two-component steady state motions (bimodal galloping) arise. However, for a certain value of $\mu$, the two-component solution disappears and the motion becomes monomodal again. Then, by increasing $\mu$, a re-stabilization of the non-trivial equilibrium path occurs. For high wind speed a further bifurcation manifests itself, in correspondence of which a bimodal galloping is triggered. A purely planar motion never occurs, due both to the steady-state forcing terms and the aerodynamic non-linearities. (3) The bimodal galloping is not always stable. When the geometric non-linearities are sufficiently large, a region of amplitude modulate motions arises, after the amplitude has undergone a Hopf bifurcation. (4) Geometrical non-linearities are very important to model the problem correctly, since they are mainiy responsible for coupling phenomena. (5) Among the aerodynamic components, the essential terms to preserve all qualitative and quantitative solution properties are the linear damping terms $\left(b_{i}, i=1,4\right)$, the cubic terms $b_{8}$ and $b_{12}$, and the steady-state forces $f_{00}$ (see equations (8)). (6) The non-linear in-plane frequency $\Omega_{2}$ always depends on in-plane oscillation amplitudes unlike classic galloping theory, mainly due to the presence of geometric non-linearities and steady-state forcing terms. (7) When non-symmetric flow occurs, the Den Hartog criterion has to be modified. An example in which such a simplistic criterion furnishes a non-protective estimation of the first galloping bifurcation has been illustrated. (8) Although the results presented here are strictly valid in internal resonance conditions, the numerical examples have shown that solutions hold in a sufficiently large region around resonance.

\section{ACKNOWLEDGMENTS}

This work has been partially supported by a MURST grant. The authors thank Prof. J. L. Lilien (Institut d'Electricité Montefiore, University of Liège) for kindly supplying non-symmetric flow experimental data.

\section{REFERENCES}

1. R. D. Blevins 1990 Flow-induced Vibration. New York: Van Nostrand Reinhold; second edition.

2. K. F. JONES 1992 American Society of Civil Engineers, Journal of Engineering Mechanics 118, 92-107. Coupled vertical and horizontal galloping.

3. P. Yu, Y. M. Desal, A. H. Shah and N. Popplewell 1993 American Society of Civil Engineers, Journal of Engineering Mechanics 119, 2404-2448. Three-degree-of-freedom model for galloping. Part I: formulation; Part II: solutions.

4. A. Luongo, G. Rega and F. Vestroni 1984 International Journal of Non-Linear Mechanics 19, 39-52. Planar non-linear free vibrations of an elastic cable.

5. C. L. LeE and N. C. Perkins 1992 Nonlinear Dynamics 3, 465-490. Nonlinear oscillations of suspended cables containing a two-to-one internal resonance.

6. A. Luongo, G. Rega and F. Vestroni 1980 Proceedings of the $V$ Italian Conference of Theoretical Applied Mechanics, Palermo, Italy 2, 225-236. Perturbation analysis of nonlinear free oscillations of a two d.o.f. suspended cable (in Italian).

7. A. LuONGo and G. PICCARDo 1995 Proceedings of the International Symposium on Cable Dynamics, Liege, Belgium, 157-164. Non-linear galloping of iced suspended cables with two-to-one internal resonance.

8. A. LuONGO and G. PICCARDO 1996 Proceedings of the 2nd European Nonlinear Oscillations Conference, Prague, Czech Republic 1, 273-276. On the influence of the torsional stiffness on non-linear galloping of suspended cables.

9. H. M. IRvine and T. K. CAUGHEY 1974 Proceedings of the Royal Society of London A341, 299-315. The linear theory of free vibrations of a suspended cables.

10. N. C. Perkins and C. D. Mote 1987 Journal of Sound and Vibration 114, 325-340. Three-dimensional vibration of travelling elastic cables. 
11. G. V. RAO and R. N. IYENGAR 1991 Journal of Sound and Vibration 149, 25-41. Internal resonance and non-linear response of a cable under periodic excitation.

12. A. Luongo and G. PicCardo 1998 Report DISAT, University of L'Aquila, L'Aquila, Italy. A nonlinear model for aeroelastic analysis of suspended cables.

13. G. PicCardo 1993 Ph. D. Thesis, University of Genoa, Genoa, Italy. Analysis of coupled aeroelastic phenomena (in Italian).

14. G. Solari 1994 in Wind-Excited Vibrations of Structures (H. Sockel, editor) 195-291. Vienna, Austria: Springer Verlag. Gust-excited vibrations.

15. G. PICCARDo 1993 Journal of Wind Engineering and Industrial Aerodynamics 48, 241-252. A methodology for the study of coupled aeroelastic phenomena.

16. G. V. Parkinson 1989 Progress in Aerospace Sciences 26, 169-224. Phenomena and modelling of flow-induced vibrations of bluff bodies.

17. M. Tunstall 1989 Proceedings of the Association des Ingénieurs Montefiore AIM, Study Day on Galloping, University of Liège, Liège, Belgium. Accretion of ice and aerodynamic coefficients.

18. M. NovaK 1969 American Society of Civil Engineers, Journal of Engineering Mechanics 95, 115-142. Aeroelastic galloping of prismatic bodies.

\section{APPENDIX A: AERODYNAMIC AND MECHANICAL COEFFICIENTS OF THE DISCRETE MODEL}

The $b_{i}, f_{n}$ aerodynamic and $c_{i}$ mechanical coefficients of the discrete model (equations (8)) are:

$$
\begin{aligned}
& b_{1}=2 \zeta_{1} \omega_{1}+\frac{\sqrt{\beta}}{\pi} c_{d} \mu, \quad b_{2}=\frac{d_{4}}{d_{11}} \frac{\sqrt{\beta}}{2 \pi}\left(c_{d}^{\prime}-c_{1}\right) \mu, \quad b_{3}=\frac{d_{4}}{d_{5}} \quad \frac{\sqrt{\beta}}{\pi} c_{1} \mu, \\
& b_{4}=2 \zeta_{2} \omega_{2}+\frac{\sqrt{\beta}}{2 \pi}\left(c_{d}+c_{l}^{\prime}\right) \mu, \quad b_{5}=-\frac{d_{12}}{d_{11}} \frac{\rho b L}{2 m} c_{d}, \quad b_{6}=-\frac{d_{8}}{d_{11}} \frac{\rho b L}{4 m}\left(c_{d}^{\prime \prime}+c_{d}-2 c_{l}^{\prime}\right), \\
& b_{7}=-\frac{d_{6}}{d_{11}} \frac{\rho b L}{2 m}\left(c_{d}^{\prime}-c_{l}\right), \quad b_{8}=\frac{d_{13}}{d_{11}}\left(\frac{\rho b L}{m}\right)^{2} \frac{\pi}{12 \sqrt{\beta}}\left(c_{d}^{\prime \prime \prime}+c_{d}^{\prime}-3 c_{l}^{\prime \prime}-3 c_{l}\right) \frac{1}{\mu}, \\
& b_{9}=-\frac{d_{6}}{d_{5}} \frac{\rho b L}{2 m} c_{l}, \quad b_{10}=-\frac{d_{7}}{d_{5}} \frac{\rho b L}{4 m}\left(c_{l}^{\prime \prime}+c_{l}+2 c_{d}^{\prime}\right) \quad b_{11}=-\frac{d_{8}}{d_{5}} \frac{\rho b L}{2 m}\left(c_{d}+c_{l}^{\prime}\right), \\
& b_{12}=\frac{d_{9}}{d_{5}}\left(\frac{\rho b L}{m}\right)^{2} \frac{\pi}{12 \sqrt{\beta}}\left(c_{l}^{\prime \prime \prime}+c_{l}^{\prime}+3 \mathrm{c}_{\mathrm{d}}^{\prime \prime}+3 \mathrm{c}_{\mathrm{d}}\right) \frac{1}{\mu}, \quad f_{10}=\frac{m}{\rho b L} \frac{\beta}{2 \pi^{2}} c_{d} \mu^{2}, \\
& f_{20}=\frac{m}{\rho b L} \frac{\beta}{2 \pi^{2}} c_{1} \mu^{2}, \quad c_{1}=-\frac{d_{1} d_{3}}{\pi^{2} d_{11}} \alpha \beta, \quad c_{2}=\frac{d_{3}^{2}}{2 \pi^{2} d_{11}} \alpha, \quad c_{3}=\frac{d_{2} d_{3}}{2 \pi^{2} d_{11}} \alpha, \\
& c_{4}=-\frac{d_{1} d_{3}}{2 \pi^{2} d_{5}} \alpha \beta, \quad c_{5}=-\frac{3 d_{1} d_{2}}{2 \pi^{2} d_{5}} \alpha \beta, \quad c_{6}=\frac{d_{2} d_{3}}{2 \pi^{2} d_{5}} \alpha, \quad c_{7}=\frac{d_{2}^{2}}{2 \pi^{2} d_{5}} \alpha
\end{aligned}
$$


Here $\zeta_{i}$ are the modal damping coefficients; $c_{j}^{\prime}, c_{j}^{\prime \prime}, c_{j}^{\prime \prime \prime}(j=d, l)$ are the first, second and third derivatives of drag $(d)$ and lift $(l)$ coefficients, with respect to the fluctuating angle of attack $\gamma_{a} ; d_{i}$ are coefficients defined as follows:

$$
\begin{gathered}
d_{1}=\int_{0}^{1} \phi_{2} \mathrm{~d} s, \quad d_{2}=\int_{0}^{1} \phi_{2}^{\prime 2} \mathrm{~d} s, \quad d_{3}=\int_{0}^{1} \phi_{1}^{\prime 2} \mathrm{~d} s, \quad d_{4}=\int_{0}^{1} \phi_{1} \phi_{2} \mathrm{~d} s, \\
d_{5}=\int_{0}^{1} \phi_{2}^{2} \mathrm{~d} s, \quad d_{6}=\int_{0}^{1} \phi_{1}^{2} \phi_{2} \mathrm{~d} s, \\
d_{7}=\int_{0}^{1} \phi_{2}^{3} \mathrm{~d} s, \quad d_{8}=\int_{0}^{1} \phi_{1} \phi_{2}^{2} \mathrm{~d} s, \quad d_{9}=\int_{0}^{1} \phi_{2}^{4} \mathrm{~d} s, \quad d_{10}=\int_{0}^{1} \phi_{1} \mathrm{~d} s, \\
d_{11}=\int_{0}^{1} \phi_{1}^{2} \mathrm{~d} s, \quad d_{12}=\int_{0}^{1} \phi_{1}^{3} \mathrm{~d} s, \quad d_{13}=\int_{0}^{1} \phi_{1} \phi_{2}^{3} \mathrm{~d} s .
\end{gathered}
$$

Here $\phi_{1}(s)$ and $\phi_{2}(s)$ are the classical vibration modal shapes taken from the linear theory [9]. In particular, the first symmetric modes are

$$
\phi_{1}(s)=\sin (\pi s), \quad \phi_{2}(s)=k_{0}[1-\tan (\pi \omega / 2) \sin (\pi \omega s)-\cos (\pi \omega s)],
$$

where $k_{0}$ is a constant chosen so that $\phi_{2}(1 / 2)=1$ and $\omega=\omega_{2} / \omega_{1}$.

\section{APPENDIX B: SMALL STATIC DISPLACEMENT SOLUTION}

The $p_{i}$ coefficients of the amplitude modulation equations (24) are

$$
\begin{gathered}
p_{1}=-\frac{1}{2} b_{1}-\frac{b_{2} \Lambda_{9}}{2}-\frac{k_{2}^{\prime} \Lambda_{8}}{2 \omega_{1}}, \\
p_{2}=-\frac{1}{2}\left[\frac{b_{7} k_{4}^{\prime}}{4 \omega_{2}}+\frac{\omega_{2} b_{7} k_{1}^{\prime}}{4 \omega_{1}^{2}}+\frac{k_{2}^{\prime} \Lambda_{11}}{2 \omega_{1}}+b_{6} \omega_{2} \Lambda_{9}+\left(b_{7} \omega_{2}+\frac{c_{1}}{\omega_{1}}\right)\left(-\frac{k_{4}^{\prime}}{8 \omega_{1} \omega_{2}}-\frac{\sigma}{4 \omega_{1}}+\frac{1}{2}\right)\right. \\
\left.+b_{5} \omega_{2} \Lambda_{2}+\frac{c_{1}^{\prime}}{2 \omega_{1}}\right], \\
p_{3}=\frac{1}{2}\left[\frac{b_{2} \Lambda_{11}}{2}+b_{6} \omega_{2} \Lambda_{8}-\frac{b_{4} b_{7}}{4}-\frac{b_{2} \omega_{2} \Lambda_{11}}{2 \omega_{1}}-b_{5} \omega_{2} \Lambda_{1}+\frac{b_{1} b_{7} \omega_{2}}{4 \omega_{1}}+\frac{b_{4}}{8 \omega_{1}}\left(b_{7} \omega_{2}+\frac{c_{1}}{\omega_{1}}\right)\right] \\
p_{4}=-\frac{b_{4}}{2}-\frac{b_{3} \Lambda_{2}}{2}-\frac{k_{3}^{\prime} \Lambda_{1}}{2 \omega_{2}}, \quad p_{5}=-\frac{3 b_{12} \omega_{2}^{2}}{8}, \\
\frac{1}{2}\left[\frac{k_{3}^{\prime} \Lambda_{3}}{2 \omega_{2}}+\left(\frac{c_{4}}{\omega_{2}}-\frac{b_{9} \omega_{1}^{2}}{\omega_{2}}\right)\left(-\frac{k_{1}^{\prime}}{4 \omega_{1} \omega_{2}}-\frac{k_{4}^{\prime}}{8 \omega_{2}^{2}}+\frac{\sigma}{4 \omega_{2}}+\frac{1}{2}\right)+\frac{c_{4}^{\prime}}{2 \omega_{2}}-\frac{b_{9} k_{1}^{\prime}}{2 \omega_{2}}-\frac{b_{11} \omega_{1}^{2} \Lambda_{9}}{2 \omega_{2}}\right], \\
p_{7}=\frac{1}{2}\left[-\frac{b_{3} \omega_{1} \Lambda_{3}}{\omega_{2}}+\frac{b_{11} \omega_{1}^{2} \Lambda_{8}}{2 \omega_{2}}+\frac{b_{1} b_{9} \omega_{1}}{2 \omega_{2}}+\left(\frac{c_{4}}{\omega_{2}}-\frac{b_{9} \omega_{1}^{2}}{\omega_{2}}\right)\left(-\frac{b_{4}}{8 \omega_{2}}+\frac{b_{1}}{4 \omega_{2}}\right)\right]
\end{gathered}
$$




$$
\begin{aligned}
p_{8}= & p_{8}^{\prime}+p_{8}^{\prime \prime}=\left[\frac{k_{4}^{\prime}}{2 \omega_{2}}-\frac{k_{4}^{\prime 2}}{8 \omega_{2}^{3}}+\frac{k_{3}^{\prime} \Lambda_{2}}{2 \omega_{2}}-\frac{b_{4}^{2}}{8 \omega_{2}}-\frac{b_{3} \Lambda_{1}}{2}+\frac{k_{4}^{\prime \prime}}{2 \omega_{2}}\right]+\left[-\frac{k_{1}^{\prime}}{\omega_{1}}-2\left(\frac{k_{1}^{\prime \prime}}{2 \omega_{1}}-\frac{b_{1}^{2}}{8 \omega_{1}}\right.\right. \\
& \left.\left.+\frac{k_{2}^{\prime} \Lambda_{9}}{2 \omega_{1}}-\frac{k_{1}^{\prime 2}}{8 \omega_{1}^{3}}-\frac{b_{2} \Lambda_{8}}{2}\right)+\sigma\right], \\
p_{9}= & p_{9}^{\prime}+p_{9}^{\prime \prime}=\left[\frac{1}{4}\left(2 b_{10} \omega_{2} \Lambda_{10}+b_{11} \omega_{2} \Lambda_{4}+\frac{c_{5} \Lambda_{10}}{\omega_{2}}+\frac{2 c_{5} \Lambda_{14}}{\omega_{2}}+\frac{3 c_{7}}{2 \omega_{2}}\right)\right]+\left\{-\frac{1}{2}\left[\frac{b_{7} \omega_{2}^{2} \Lambda_{5}}{2 \omega_{1}}\right.\right. \\
& +\frac{c_{1} \Lambda_{5}}{2 \omega_{1}}+\frac{b_{6} \omega_{2}^{2} \Lambda_{11}}{\omega_{1}}+b_{6} \omega_{2} \Lambda_{12}+\frac{c_{3}}{\omega_{1}}+\frac{b_{7} \omega_{2}}{4 \omega_{1}}\left(b_{7} \omega_{2}+\frac{c_{1}}{\omega_{1}}\right)+\frac{b_{7} \omega_{2} \Lambda_{5}}{2} \\
& \left.\left.-b_{6} \omega_{2} \Lambda_{11}+\frac{1}{8 \omega_{1}}\left(b_{7} \omega_{2}+\frac{c_{1}}{\omega_{1}}\right)^{2}+\frac{c_{1} \Lambda_{14}}{\omega_{1}}+\frac{b_{6} \omega_{2}^{2} \Lambda_{12}}{\omega_{1}}\right]\right\} \\
p_{10}= & p_{10}^{\prime}+p_{10}^{\prime \prime}=\left\{\frac { 1 } { 4 } \left[\frac{b_{11} \omega_{1} \Lambda_{12}}{2}-\frac{1}{4 \omega_{2}}\left(b_{7} \omega_{2}+\frac{c_{1}}{\omega_{1}}\right)\left(\frac{c_{4}}{\omega_{2}}-\frac{b_{9} \omega_{1}^{2}}{\omega_{2}}\right)+\frac{b_{11} \omega_{1}^{2} \Lambda_{12}}{2 \omega_{2}}+\frac{c_{4} \Lambda_{5}}{\omega_{2}}\right.\right. \\
& +\frac{2 c_{5} \Lambda_{13}}{\omega_{2}}+\frac{b_{11} \omega_{1}^{2} \Lambda_{11}}{2 \omega_{2}}+\frac{c_{6}}{\omega_{2}}+\frac{b_{9} \omega_{1}^{2} \Lambda_{5}}{\omega_{2}}-\frac{b_{9} \omega_{1}}{2 \omega_{2}}\left(b_{7} \omega_{2}+\frac{c_{1}}{\omega_{1}}\right)+b_{9} \omega_{1} \Lambda_{5} \\
& \left.\left.-\frac{b_{11} \omega_{1} \Lambda_{11}}{2}\right]\right\}+\left\{-\frac{1}{2}\left[2 b_{5} \omega_{1} \Lambda_{3}+\frac{b_{7}}{4}\left(\frac{c_{4}}{\omega_{2}}-\frac{b_{9} \omega_{1}^{2}}{\omega_{2}}\right)+\frac{c_{1} \Lambda_{13}}{\omega_{1}}+\frac{3 c_{2}}{2 \omega_{1}}\right.\right. \\
& \left.\left.-\frac{1}{8 \omega_{1}}\left(b_{7} \omega_{2}+\frac{c_{1}}{\omega_{1}}\right)\left(\frac{c_{4}}{\omega_{2}}-\frac{b_{9} \omega_{1}^{2}}{\omega_{2}}\right)\right]\right\} .
\end{aligned}
$$

Here the $\Lambda_{i}$ coefficients have the expressions

$$
\begin{gathered}
\Lambda_{1}=-\frac{\omega_{2}}{\omega_{1}^{2}-\omega_{2}^{2}} b_{2}, \quad \Lambda_{2}=-\frac{k_{2}^{\prime}}{\omega_{1}^{2}-\omega_{2}^{2}}, \quad \Lambda_{3}=-\frac{b_{5}}{3}, \quad \Lambda_{4}=\frac{b_{6} \omega_{2}^{2}}{\omega_{1}^{2}-4 \omega_{2}^{2}}, \\
\Lambda_{5}=\frac{c_{1}-b_{7} \omega_{1} \omega_{2}}{\omega_{2}^{2}+2 \omega_{1} \omega_{2}}, \quad \Lambda_{6}=-b_{5}, \quad \Lambda_{7}=-\frac{\omega_{2}^{2}}{\omega_{1}^{2}} b_{6}, \quad \Lambda_{8}=-\frac{b_{3} \omega_{1}}{\omega_{2}^{2}-\omega_{1}^{2}}, \\
\Lambda_{9}=-\frac{k_{3}^{\prime}}{\omega_{2}^{2}-\omega_{1}^{2}}, \quad \Lambda_{10}=\frac{c_{5}-b_{10} \omega_{2}^{2}}{3 \omega_{2}^{2}}, \quad \Lambda_{11}=\frac{b_{11} \omega_{1} \omega_{2}}{\omega_{1}^{2}-2 \omega_{1} \omega_{2}}, \\
\Lambda_{12}=-\frac{b_{11} \omega_{1} \omega_{2}}{\omega_{1}^{2}+2 \omega_{1} \omega_{2}}, \quad \Lambda_{13}=-\frac{c_{4}+b_{9} \omega_{1}^{2}}{\omega_{2}^{2}}, \quad \Lambda_{14}=-\frac{c_{5}+b_{10} \omega_{2}^{2}}{\omega_{2}^{2}} .
\end{gathered}
$$

\section{APPENDIX C: LARGE STATIC DISPLACEMENT SOLUTION}

The $r_{i}$ and $s_{i}$ coefficients of the equations of motion (26) are

$$
\begin{gathered}
s_{1}=\left(-h_{1}-\alpha_{1}^{2} h_{2}-\alpha_{1} c_{1}^{*}+\alpha_{1}^{2} \alpha_{2} c_{5}^{*}+\alpha_{2} c_{4}^{*}\right) / k, \\
s_{2}=\left(-c_{1}^{*}-\alpha_{1} \alpha_{2} c_{1}^{*}-2 \alpha_{1} h_{2}-2 \alpha_{2} h_{1}+2 \alpha_{2}^{2} c_{4}^{*}+2 \alpha_{1} \alpha_{2} c_{5}^{*}\right) / k,
\end{gathered}
$$




$$
\begin{aligned}
& s_{3}=\left(-h_{2}-\alpha_{2} c_{1}^{*}-\alpha_{2}^{2} h_{1}+\alpha_{2}^{3} c_{4}^{*}+\alpha_{2} c_{5}^{*}\right) / k, \\
& s_{4}=\left(-c_{2}+\alpha_{1}^{2} \alpha_{2} h_{3}+\alpha_{1} \alpha_{2} c_{6}+\alpha_{1}^{3} \alpha_{2} c_{7}-\alpha_{1}^{2} c_{3}\right) / k, \\
& s_{5}=\left(2 \alpha_{1} \alpha_{2} h_{3}+3 \alpha_{1}^{2} \alpha_{2} c_{7}+\alpha_{2} c_{6}+\alpha_{1}^{2} \alpha_{2}^{2} h_{3}+2 \alpha_{1} \alpha_{2}^{2} c_{6}-3 \alpha_{2} c_{2}-2 \alpha_{1} c_{3}-\alpha_{1}^{2} \alpha_{2} c_{3}\right) / k \text {, } \\
& s_{6}=\left(-c_{3}+3 \alpha_{1} \alpha_{2} c_{7}+\alpha_{2} h_{3}+2 \alpha_{1} \alpha_{2}^{2} h_{3}+2 \alpha_{2}^{2} c_{6}+\alpha_{1} \alpha_{2}^{3} c_{6}-3 \alpha_{2}^{2} c_{2}-2 \alpha_{1} \alpha_{2} c_{3}\right) / k \text {, } \\
& s_{7}=\left(\alpha_{2} c_{7}+\alpha_{2}^{2} h_{3}+\alpha_{2}^{3} c_{6}-\alpha_{2}^{3} c_{2}-\alpha_{2} c_{3}\right) / k \text {, } \\
& s_{8}=\left(-c_{4}^{*}-\alpha_{1}^{2} c_{5}^{*}+\alpha_{1}^{2} c_{1}^{*}+\alpha_{1} h_{1}+\alpha_{1}^{3} h_{2}\right) / k \text {, } \\
& s_{9}=\left(\alpha_{1}^{2} \alpha_{2} c_{1}^{*}-2 \alpha_{1} c_{5}^{*}-2 \alpha_{2} c_{4}^{*}+\alpha_{1} c_{1}^{*}+2 \alpha_{1} \alpha_{2} h_{1}+2 \alpha_{1}^{2} h_{2}\right) / k \text {, } \\
& s_{10}=\left(-c_{5}^{*}+\alpha_{1} \alpha_{2}^{2} h_{1}+\alpha_{1} h_{2}-\alpha_{2}^{2} c_{4}^{*}+\alpha_{1} \alpha_{2} c_{*}^{*}\right) / k \text {, } \\
& s_{11}=\left(-\alpha_{1} c_{6}+\alpha_{1} c_{2}+\alpha_{1}^{3} c_{3}-\alpha_{1}^{2} h_{3}-\alpha_{1}^{3} c_{7}\right) / k \text {, } \\
& s_{12}=\left(-c_{6}-\alpha_{1}^{2} \alpha_{2} h_{3}+2 \alpha_{1}^{2} c_{3}-2 \alpha_{1} h_{3}-2 \alpha_{1} \alpha_{2} c_{6}-3 \alpha_{1}^{2} c_{7}+3 \alpha_{1} \alpha_{2} c_{2}+\alpha_{1}^{3} \alpha_{2} c_{3}\right) / k \text {, } \\
& s_{13}=\left(-h_{3}+3 \alpha_{1} \alpha_{2}^{2} c_{2}-2 \alpha_{1} \alpha_{2} h_{3}+\alpha_{1} c_{3}+2 \alpha_{1}^{2} \alpha_{2} c_{3}-\alpha_{1} \alpha_{2}^{2} c_{6}-2 \alpha_{2} c_{6}-3 \alpha_{1} c_{7}\right) / k \text {, } \\
& s_{14}=\left(-c_{7}-\alpha_{2}^{2} c_{6}+\alpha_{1} \alpha_{2}^{3} c_{2}+\alpha_{1} \alpha_{2} c_{3}-\alpha_{2} h_{3}\right) / k \text {, } \\
& r_{1}=\left(-b_{1}-\alpha_{1} b_{2}+\alpha_{1} \alpha_{2} b_{4}+\alpha_{2} b_{3}\right) / k, \quad r_{2}=\left(-b_{2}-\alpha_{2} b_{1}+\alpha_{2}^{2} b_{3}+\alpha_{2} b_{4}\right) / k, \\
& r_{3}=\left(-b_{3}+\alpha_{1} b_{1}+\alpha_{1}^{2} b_{2}-\alpha_{1} b_{4}\right) / k, \quad r_{4}=\left(-b_{4}-\alpha_{2} b_{3}+\alpha_{1} \alpha_{2} b_{1}+\alpha_{1} b_{2}\right) / k, \\
& r_{5}=\left(-b_{5}+\alpha_{1}^{2} \alpha_{2} b_{10}+\alpha_{1} \alpha_{2} b_{11}+\alpha_{2} b_{9}-\alpha_{1}^{2} b_{6}-\alpha_{1} b_{7}\right) / \mathrm{k}, \\
& r_{6}=\left(-b_{7}+2 \alpha_{1} \alpha_{2} b_{10}+\alpha_{2} b_{11}+2 \alpha_{2}^{2} b_{9}+\alpha_{1} \alpha_{2}^{2} b_{11}-\alpha_{1} \alpha_{2} b_{7}-2 \alpha_{2} b_{5}-2 \alpha_{1} b_{6}\right) / k \text {, } \\
& r_{7}=\left(-b_{6}-\alpha_{2}^{2} b_{5}+\alpha_{2} b_{10}+\alpha_{2}^{3} b_{9}+\alpha_{2}^{2} b_{11}-\alpha_{2} b_{7}\right) / k, \quad r_{8}=\left(\alpha_{1}^{3} \alpha_{2} b_{12}-\alpha_{1}^{3} b_{8}\right) / k \text {, } \\
& r_{9}=\left(3 \alpha_{1}^{2} \alpha_{2} b_{12}-3 \alpha_{1}^{2} b_{8}\right) / k, \quad r_{10}=\left(3 \alpha_{1} \alpha_{2} b_{12}-3 \alpha_{1} b_{8}\right) / k, \quad r_{11}=\left(-b_{8}+\alpha_{2} b_{12}\right) / k \text {, } \\
& r_{12}=\left(-b_{9}+\alpha_{1}^{2} b_{7}+\alpha_{1}^{3} b_{6}-\alpha_{1}^{2} b_{10}-\alpha_{1} b_{11}+\alpha_{1} b_{5}\right) / \mathrm{k} \text {, } \\
& r_{13}=\left(-b_{11}+\alpha_{1} b_{7}-\alpha_{1} \alpha_{2} b_{11}+2 \alpha_{1} \alpha_{2} b_{5}-2 \alpha_{2} b_{9}+\alpha_{1}^{2} \alpha_{2} b_{7}+2 \alpha_{1}^{2} b_{6}-2 \alpha_{1} b_{10}\right) / k \text {, } \\
& r_{14}=\left(-b_{10}+\alpha_{1} b_{6}-\alpha_{2} b_{11}+\alpha_{1} \alpha_{2} b_{7}-\alpha_{2}^{2} b_{9}+\alpha_{1} \alpha_{2}^{2} b_{5}\right) / k, \\
& r_{15}=\left(\alpha_{1}^{4} b_{8}-\alpha_{1}^{3} b_{12}\right) / k . \quad r_{16}=\left(3 \alpha_{1}^{3} b_{8}-3 \alpha_{1}^{2} b_{12}\right) / k, \quad r_{17}=\left(3 \alpha_{1}^{2} b_{8}-3 \alpha_{1} b_{12}\right) / k \text {, } \\
& r_{18}=\left(-b_{12}+\alpha_{1} b_{8}\right) / k \text {. }
\end{aligned}
$$

Here

$$
\begin{gathered}
k=\alpha_{1} \alpha_{2}-1, \quad \alpha_{1}=-\frac{k_{3}}{k_{4}-\tilde{\omega}_{1}^{2}}, \quad \alpha_{2}=-\frac{k_{4}-\tilde{\omega}_{2}^{2}}{k_{3}}, \\
\tilde{\omega}_{1.2}^{2}=\frac{\left(k_{1}+k_{4}\right) \mp \sqrt{\left(k_{1}+k_{4}\right)^{2}-4\left(k_{1} k_{4}-k_{2} k_{3}\right)}}{2} .
\end{gathered}
$$

The $p_{i}$ coefficients of the amplitude modulation equations (27) are

$$
\begin{gathered}
p_{1}=-\frac{1}{2} r_{1}, \quad p_{2}=-\frac{1}{2}\left(r_{6} \tilde{\omega}_{2}+\frac{s_{2}}{\tilde{\omega}_{1}}\right)\left(-\frac{\sigma}{4 \tilde{\omega}_{1}}+\frac{1}{2}\right), \\
p_{3}=\frac{1}{2}\left[-r_{5} \tilde{\omega}_{2} \Lambda_{1}+r_{7} \tilde{\omega}_{2} \Lambda_{8}-\frac{r_{2} \tilde{\omega}_{2} \Lambda_{11}}{2 \tilde{\omega}_{1}}-\frac{s_{1} \Lambda_{1}}{\tilde{\omega}_{1}}-\frac{r_{4} r_{6}}{4}+\frac{r_{2} \Lambda_{11}}{2}+\frac{r_{1} r_{6} \tilde{\omega}_{2}}{4 \tilde{\omega}_{1}}+\frac{s_{3} \Lambda_{8}}{\tilde{\omega}_{1}}\right. \\
\left.+\frac{r_{4}}{8 \tilde{\omega}_{1}}\left(r_{6} \tilde{\omega}_{2}+\frac{s_{2}}{\tilde{\omega}_{1}}\right)\right],
\end{gathered}
$$




$$
\begin{aligned}
& p_{4}=-\frac{1}{2} r_{4}, \quad p_{5}=-\frac{3}{8} r_{18} \tilde{\omega}_{2}^{2}, \quad p_{6}=\frac{1}{2}\left(\frac{s_{8}}{\tilde{\omega}_{2}}-\frac{r_{12} \tilde{\omega}_{1}^{2}}{\tilde{\omega}_{2}}\right)\left(\frac{\sigma}{4 \tilde{\omega}_{2}}+\frac{1}{2}\right) \\
& p_{7}=\frac{1}{2}\left[\frac{r_{13} \tilde{\omega}_{1}^{2} \Lambda_{8}}{2 \tilde{\omega}_{2}}+\frac{r_{1} r_{12} \tilde{\omega}_{1}}{2 \tilde{\omega}_{2}}-\frac{r_{3} \tilde{\omega}_{1} \Lambda_{3}}{\tilde{\omega}_{2}}+\left(\frac{s_{8}}{\tilde{\omega}_{2}}-\frac{r_{12} \tilde{\omega}_{1}^{2}}{\tilde{\omega}_{2}}\right)\left(-\frac{r_{4}}{8 \tilde{\omega}_{2}}+\frac{r_{1}}{4 \tilde{\omega}_{2}}\right)-\frac{s_{9} \Lambda_{8}}{2 \tilde{\omega}_{2}}\right], \\
& p_{8}=p_{8}^{\prime}+p_{8}^{\prime \prime}=\left[\frac{1}{8 \tilde{\omega}_{2}}\left(-r_{4}^{2}-4 r_{3} \tilde{\omega}_{2} \Lambda_{1}\right)\right]+\left[-\frac{1}{4 \tilde{\omega}_{1}}\left(-r_{1}^{2}-4 r_{2} \tilde{\omega}_{1} \Lambda_{8}\right)+\sigma\right] \\
& p_{9}=p_{9}^{\prime}+p_{9}^{\prime \prime}=\left[\frac{1}{4}\left(\frac{s_{10} \Lambda_{10}}{\tilde{\omega}_{2}}+\frac{s_{9} \Lambda_{4}}{2 \tilde{\omega}_{2}}+\frac{3 s_{14}}{2 \tilde{\omega}_{2}}+2 r_{14} \tilde{\omega}_{2} \Lambda_{10}+\frac{2 s_{10} \Lambda_{14}}{\tilde{\omega}_{2}}+r_{13} \tilde{\omega}_{2} \Lambda_{4}+\frac{s_{9} \Lambda_{7}}{\tilde{\omega}_{2}}\right)\right] \\
& +\left\{-\frac{1}{2}\left[\frac{1}{8 \tilde{\omega}_{1}}\left(r_{6} \tilde{\omega}_{2}+\frac{s_{2}}{\tilde{\omega}_{1}}\right)^{2}-r_{7} \tilde{\omega}_{2} \Lambda_{11}+\frac{s_{3} \Lambda_{12}}{\tilde{\omega}_{1}}+r_{7} \tilde{\omega}_{2} \Lambda_{12}+\frac{s_{2} \Lambda_{5}}{2 \tilde{\omega}_{1}}\right.\right. \\
& +\frac{r_{6} \tilde{\omega}_{2}}{4 \tilde{\omega}_{1}}\left(r_{6} \tilde{\omega}_{2}+\frac{s_{2}}{\tilde{\omega}_{1}}\right)+\frac{2 s_{1} \Lambda_{7}}{\tilde{\omega}_{1}}+\frac{r_{7} \tilde{\omega}_{2}^{2} \Lambda_{12}}{\tilde{\omega}_{1}}+\frac{s_{2} \Lambda_{14}}{\tilde{\omega}_{1}}+\frac{r_{6} \tilde{\omega}_{2} \Lambda_{5}}{2}+\frac{s_{3} \Lambda_{11}}{\tilde{\omega}_{1}} \\
& \left.\left.+\frac{s_{6}}{\tilde{\omega}_{1}}+\frac{r_{6} \tilde{\omega}_{2}^{2} \Lambda_{5}}{2 \tilde{\omega}_{1}}+\frac{r_{7} \tilde{\omega}_{2}^{2} \Lambda_{11}}{\tilde{\omega}_{1}}\right]\right\} \\
& p_{10}=p_{10}^{\prime}+p_{10}^{\prime \prime}=\left\{\frac { 1 } { 4 } \left[\frac{s_{12}}{\tilde{\omega}_{2}}-\frac{r_{12} \tilde{\omega}_{1}}{2 \tilde{\omega}_{2}}\left(r_{6} \tilde{\omega}_{2}+\frac{s_{2}}{\tilde{\omega}_{1}}\right)+\frac{r_{12} \tilde{\omega}_{1}^{2} \Lambda_{5}}{\tilde{\omega}_{2}}+\frac{r_{13} \tilde{\omega}_{1}^{2} \Lambda_{11}}{2 \tilde{\omega}_{2}}+\frac{s_{8} \Lambda_{5}}{\tilde{\omega}_{2}}+\frac{r_{13} \tilde{\omega}_{1}^{2} \Lambda_{12}}{2 \tilde{\omega}_{2}}\right.\right. \\
& -\frac{r_{13} \tilde{\omega}_{1} \Lambda_{11}}{2}-\frac{1}{4 \tilde{\omega}_{2}}\left(r_{6} \tilde{\omega}_{2}+\frac{s_{2}}{\tilde{\omega}_{1}}\right)\left(\frac{s_{8}}{\tilde{\omega}_{2}}-r_{12} \frac{\tilde{\omega}_{1}^{2}}{\tilde{\omega}_{2}}\right)+\frac{s_{9} \Lambda_{11}}{2 \tilde{\omega}_{2}}+\frac{s_{9} \Lambda_{6}}{\tilde{\omega}_{2}}+r_{12} \tilde{\omega}_{1} \Lambda_{5} \\
& \left.\left.+\frac{s_{9} \Lambda_{12}}{2 \tilde{\omega}_{2}}+\frac{2 s_{10} \Lambda_{13}}{\tilde{\omega}_{2}}+\frac{r_{13} \tilde{\omega}_{1} \Lambda_{12}}{2}\right]\right\}+\left\{-\frac{1}{2}\left[\frac{s_{2} \Lambda_{13}}{\tilde{\omega}_{1}}-\frac{1}{8 \tilde{\omega}_{1}}\left(r_{6} \tilde{\omega}_{2}+\frac{s_{2}}{\tilde{\omega}_{1}}\right)\right.\right. \\
& \left.\left.\cdot\left(\frac{s_{8}}{\tilde{\omega}_{2}}-r_{12} \frac{\tilde{\omega}_{1}^{2}}{\tilde{\omega}_{2}}\right)+\frac{s_{1} \Lambda_{3}}{\tilde{\omega}_{1}}+\frac{r_{6}}{4}\left(\frac{s_{8}}{\tilde{\omega}_{2}}-r_{12} \frac{\tilde{\omega}_{1}^{2}}{\tilde{\omega}_{2}}\right)+\frac{2 s_{1} \Lambda_{6}}{\tilde{\omega}_{1}}+2 r_{5} \tilde{\omega}_{1} \Lambda_{3}+\frac{3 s_{4}}{2 \tilde{\omega}_{1}}\right]\right\}, \\
& p_{11}=-\frac{3}{8} r_{8} \tilde{\omega}_{1}^{2}, \quad p_{12}=-\frac{r_{10} \tilde{\omega}_{2}^{2}}{4}, \quad p_{13}=-\frac{r_{16} \tilde{\omega}_{1}^{2}}{4} .
\end{aligned}
$$

Here the $\Lambda_{i}$ coefficients have the expressions

$$
\begin{gathered}
\Lambda_{1}=-\frac{\tilde{\omega}_{2}}{\tilde{\omega}_{1}^{2}-\tilde{\omega}_{2}^{2}} r_{2}, \quad \Lambda_{2}=0, \quad \Lambda_{3}=\frac{s_{1}-r_{5} \tilde{\omega}_{1}^{2}}{3 \tilde{\omega}_{1}^{2}}, \quad \Lambda_{4}=-\frac{s_{3}-r_{7} \tilde{\omega}_{2}^{2}}{\tilde{\omega}_{1}^{2}-4 \tilde{\omega}_{2}^{2}}, \\
\Lambda_{5}=\frac{s_{2}-r_{6} \tilde{\omega}_{1} \tilde{\omega}_{2}}{\tilde{\omega}_{2}^{2}+2 \tilde{\omega}_{1} \tilde{\omega}_{2}}, \quad \Lambda_{6}=-\frac{s_{1}+r_{5} \tilde{\omega}_{1}^{2}}{\tilde{\omega}_{1}^{2}}, \quad \Lambda_{7}=-\frac{s_{3}+r_{7} \tilde{\omega}_{2}^{2}}{\tilde{\omega}_{1}^{2}}, \quad \Lambda_{8}=-\frac{r_{3} \tilde{\omega}_{1}}{\tilde{\omega}_{2}^{2}-\tilde{\omega}_{1}^{2}}, \\
\Lambda_{9}=0, \quad \Lambda_{10}=\frac{s_{10}-r_{14} \tilde{\omega}_{2}^{2}}{3 \tilde{\omega}_{2}^{2}}, \quad \Lambda_{11}=\frac{s_{9}+r_{13} \tilde{\omega}_{1} \tilde{\omega}_{2}}{\tilde{\omega}_{1}^{2}-2 \tilde{\omega}_{1} \tilde{\omega}_{2}}, \quad \Lambda_{12}=\frac{s_{9}-r_{13} \tilde{\omega}_{1} \tilde{\omega}_{2}}{\tilde{\omega}_{1}^{2}+2 \tilde{\omega}_{1} \tilde{\omega}_{2}}, \\
\Lambda_{13}=-\frac{s_{8}+r_{12} \tilde{\omega}_{1}^{2}}{\tilde{\omega}_{2}^{2}}, \quad \Lambda_{14}=-\frac{s_{10}+r_{14} \tilde{\omega}_{2}^{2}}{\tilde{\omega}_{2}^{2}} .
\end{gathered}
$$

